\title{
Toward Supersonic Retropropulsion CFD Validation
}

\author{
Bil Kleb* $\quad$ D. Guy Schauerhamer ${ }^{\dagger} \quad$ Kerry Trumble $^{\ddagger}$ \\ NASA Langley, Hampton, VA Jacobs, Houston, TX NASA Ames, Moffett Field, CA \\ Emre Sozer ${ }^{\S}$ and Michael Barnhardt ${ }^{\ddagger} \quad$ Jan-Reneé Carlson ${ }^{\llbracket}$ and Karl Edquist ${ }^{\|}$ \\ ERC, Moffett Field, CA NASA Langley, Hampton, VA
}

This paper begins the process of verifying and validating computational fluid dynamics (CFD) codes for supersonic retropropulsive flows. Four CFD codes (DPLR, FUN3D, OVERFLOW, and US3D) are used to perform various numerical and physical modeling studies toward the goal of comparing predictions with a wind tunnel experiment specifically designed to support CFD validation. Numerical studies run the gamut in rigor from code-to-code comparisons to observed order-of-accuracy tests. Results indicate that this complex flowfield, involving time-dependent shocks and vortex shedding, design order of accuracy is not clearly evident. Also explored is the extent of physical modeling necessary to predict the salient flowfield features found in high-speed Schlieren images and surface pressure measurements taken during the validation experiment. Physical modeling studies include geometric items such as wind tunnel wall and sting mount interference, as well as turbulence modeling that ranges from a RANS (Reynolds-Averaged Navier-Stokes) 2equation model to DES (Detached Eddy Simulation) models. These studies indicate that tunnel wall interference is minimal for the cases investigated; model mounting hardware effects are confined to the aft end of the model; and sparse grid resolution and turbulence modeling can damp or entirely dissipate the unsteadiness of this self-excited flow.

\section{Nomenclature}

$\begin{array}{llll}\alpha & \text { Angle of attack } & m & \text { Slope } \\ \Delta t & \text { Time step } & N & \text { Number of grid nodes } \\ \dot{m} & \text { mass flow rate } & p_{0, \text { jet }} & \text { Jet stagnation pressure } \\ \theta & \text { Angle measured from top of model } & p_{\infty} & \text { Freestream static pressure } \\ & \text { anti-clockwise when viewed along the } & R & \text { Model radius, } 2.5^{\prime \prime} \\ & \text { positive } x \text { direction } & r & \text { Radius from } x \text { axis } \\ C_{A} & \text { Coefficient of axial force } & R^{2} & \text { Coefficient of determination } \\ c_{p} & \text { Coefficient of pressure } & R e & \text { Reynolds number } \\ C_{D} & \text { Coefficient of drag } & T_{0, \text { jet }} & \text { Jet stagnation temperature } \\ C_{L} & \text { Coefficient of lift } & T_{\infty} & \text { Freestream static temperature } \\ C_{T} & \text { Coefficient of thrust } & x & \text { Distance from spherical nose cap } \\ D & \text { Model diameter, } 5^{\prime \prime} & & \text { along model axis } \\ f & \text { Frequency } & y & \text { Distance from model axis out the } \\ h & \text { Mesh spacing } & & \text { starboard side } \\ L & \text { Model extent in } x \text {-direction, } 10.5^{\prime \prime} & z & \text { Distance from model axis upward }\end{array}$

\footnotetext{
*Aerospace Engineer, Aerothermodynamics Branch, MS 408A, Lifetime Member.

$\dagger$ Aerospace Engineer, Applied Aerosciences and CFD Branch, MS EG-3, Member.

${ }^{\ddagger}$ Research Scientist, Aerothermodynamics Branch, MS 230-2, Senior Member.

$\S$ Research Scientist, Aerothermodynamics Branch, MS 230-2, Member.

ף Aerospace Engineer, Computational Aero-Sciences Branch, MS 128, Senior Member.

" Aerospace Engineer, Atmospheric Flight and Entry Systems Branch, MS 489, Senior Member.
} 


\section{Introduction}

Future Mars missions will demand entry, descent, and landing (EDL) performance beyond the capabilities of current parachute-based deceleration systems due to much higher payload masses required for human exploration. One alternate deceleration technique is the use of propulsive deceleration during the supersonic phase of the entry trajectory as opposed to merely the terminal-landing phase. This supersonic retropropulsion (SRP), or the use of engine thrust directed into the oncoming supersonic freestream flow, has become a focus of the EDL community. ${ }^{1-5}$

Because Earth-based testing facilities cannot simultaneously simulate all Mars flight parameters, a portion of the Mars entry aerodynamic database will have to come from CFD prediction. The nuclear power and weapons communities have long faced a similar circumstance: a lack of, or outright ban on, experimental testing. They have spent decades researching the verification, validation, and uncertainty quantification steps necessary to build confidence in their numerical predictions. ${ }^{6}$

This trend towards flight vehicle design based solely on CFD is discussed by Aeschliman and Oberkampf, ${ }^{7}$ who also describe a systematic approach for designing CFD validation experiments. To date, only a handful of ground-based retropropulsion experiments have been conducted, ${ }^{8-10}$ and they were not designed for CFD validation as revealed by recent attempts to simulate them with CFD. ${ }^{11-15}$ Aeschliman and Oberkampf warned of these difficulties:

We consider unsatisfactory the common practice of attempting to validate codes using published data obtained for some purpose unrelated to code validation. Almost inevitably, critical information required by the code, boundary and initial conditions especially, will be unavailable.

To rectify this, a CFD validation experiment has been designed, ${ }^{16}$ phase I testing has been conducted in the NASA Langley $4^{\prime} \times 4^{\prime}$ Unitary Plan Wind Tunnel (UPWT), ${ }^{17}$ and phase II, an entry in the NASA Ames $9^{\prime} \times 7^{\prime}$ Supersonic Wind Tunnel, is scheduled to be complete by the end of 2011. While the rigorous uncertainty quantification analysis has yet to be completed for phase I, initial results are available for comparison and will be revealed toward the end of this paper. The bulk of this paper, however, chronicles many of the numerical verification and physical modeling studies done to prepare for predicting the results from this CFD validation experiment and goes beyond those documented during the design of the CFD validation experiment itself. ${ }^{16,18}$

The following section contains a brief description of the validation experiment and the case chosen as the initial focus for comparison. Next, each of the four CFD codes employed, DPLR, ${ }^{19}$ Fun3D, ${ }^{20,21}$ OverFLOW, ${ }^{22}$ and Us3D, ${ }^{23}$ are discussed along with code-specific verification, solution verification, and numerical sensitivity studies. Afterward, several modeling effects are considered: wind tunnel wall interference, sting effects, and turbulence modeling. Finally, CFD predictions are assessed against the preliminary experimental data and followed by concluding remarks. 


\section{Validation Experiment Case Considered}

The $5^{\prime \prime}$-diameter, 70-degree sphere-cone-cylinder wind tunnel model as mounted in test section 2 of the NASA Langley UPWT,${ }^{24}$ is shown in Figure 1 while the surface pressure instrumentation layout is shown in Figure 2. Thanks to an interchangeable nozzle-plug design, the model can have a variety of configurations.

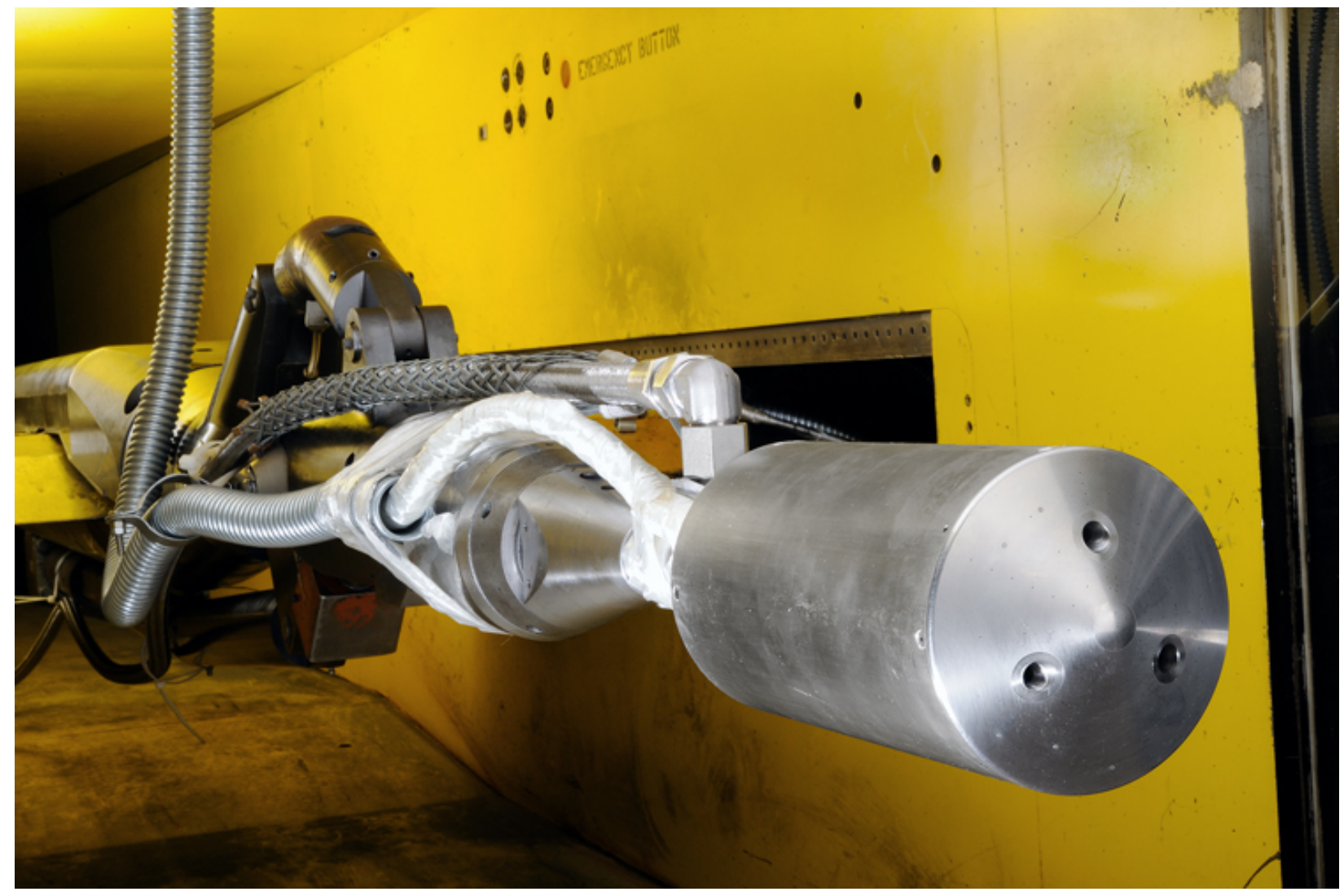

Figure 1: Mounting apparatus and the $5^{\prime \prime}$-diameter model with 3 nozzles as installed in test section 2 of the NASA Langley Unitary Plan Wind Tunnel. ${ }^{24}$

During the phase I test campaign, 0-, 1-, 3-, and 4-jet configurations were tested at Mach 2.4, 3.5, and 4.6 with angles of attack ranging from $-8^{\circ}$ to $20^{\circ}$ and Reynolds number per foot of $1.5 \mathrm{M}$ for Mach 4.6 and $1 \mathrm{M}$ for Machs 2.4 and 3.5. Nominal jet thrust coefficients, $C_{T}$, spanned 0.5 to 3 with isolated runs as high as 6 . For more details of the experiment, please refer to the companion paper by Berry et al. ${ }^{17}$

The experimental run chosen for comparison in this paper, Run 165, represents the simplest jet-on case available in that it has a quasi-periodic flowfield structure in which a ring vortex at the tripoint of the jet-plume barrel shock, a shear layer formed by large entrained recirculation region, and the jet termination shock is shed periodically. The zero angle-of-attack point of this run has the additional, simplifying advantage of being largely axisymmetric and almost perfectly periodic with a frequency around $2 \mathrm{kHz}$.

Run 165 freestream conditions are Mach 4.6, 1.5M Re/ft, and $65 \mathrm{~K}$ air with a nozzle pressure ratio, $p_{0, \text { jet }} / p_{\infty}$, of 7,724 and a nozzle temperature ratio, $T_{0, \text { jet }} / T_{\infty}$, of 5.34 . For the $4: 1$ area ratio conical nozzles, these conditions produce a thrust coefficient, $C_{T}$, of 1.97 and a mass flow rate, $\dot{m}$, of $0.62 \mathrm{lbm} / \mathrm{s}$.

Figure 3 on page 5 shows tableaux of high-speed Schlieren movie still frames for $0^{\circ}$ and $12^{\circ}$ angle of attack. If viewed in electronic form with a viewer that supports embedded Adobe Shockwave ${ }^{\text {TM }}$ Flash movies, selecting a tableau will play the corresponding movie from which the stills were extracted. To exit a movie, select it and press the escape key. 


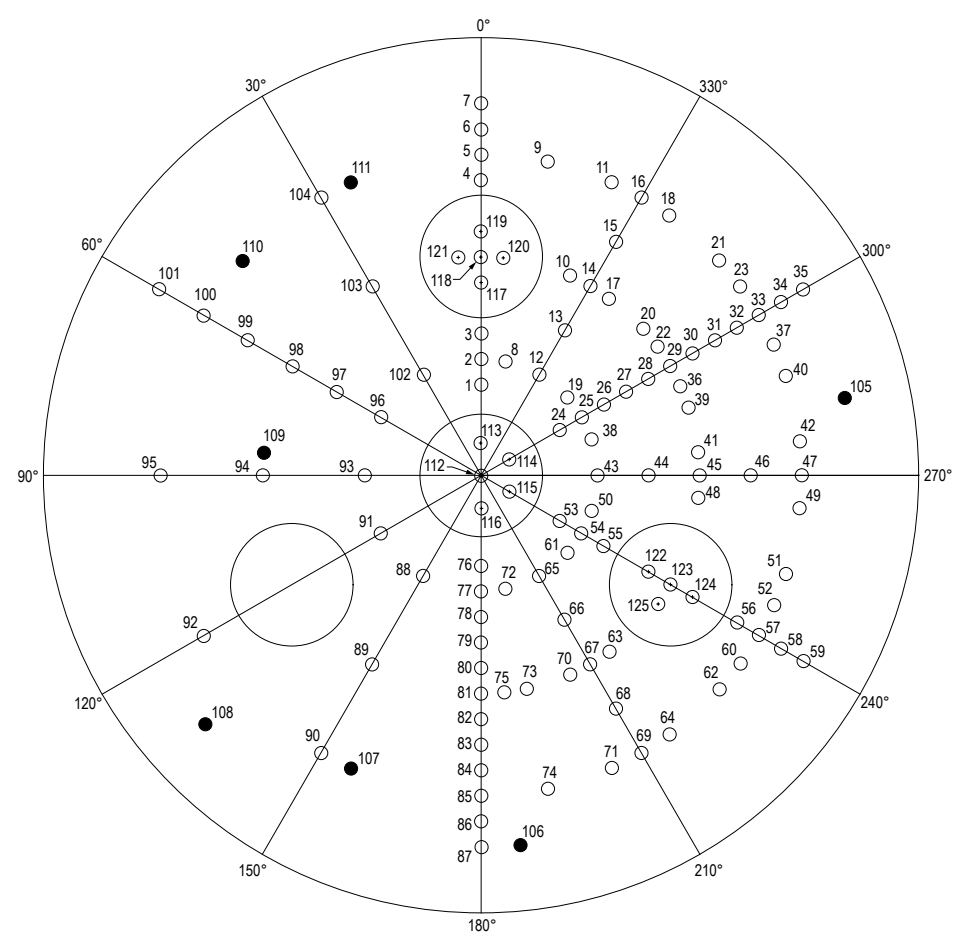

(a) Forebody as viewed from the front of the model.

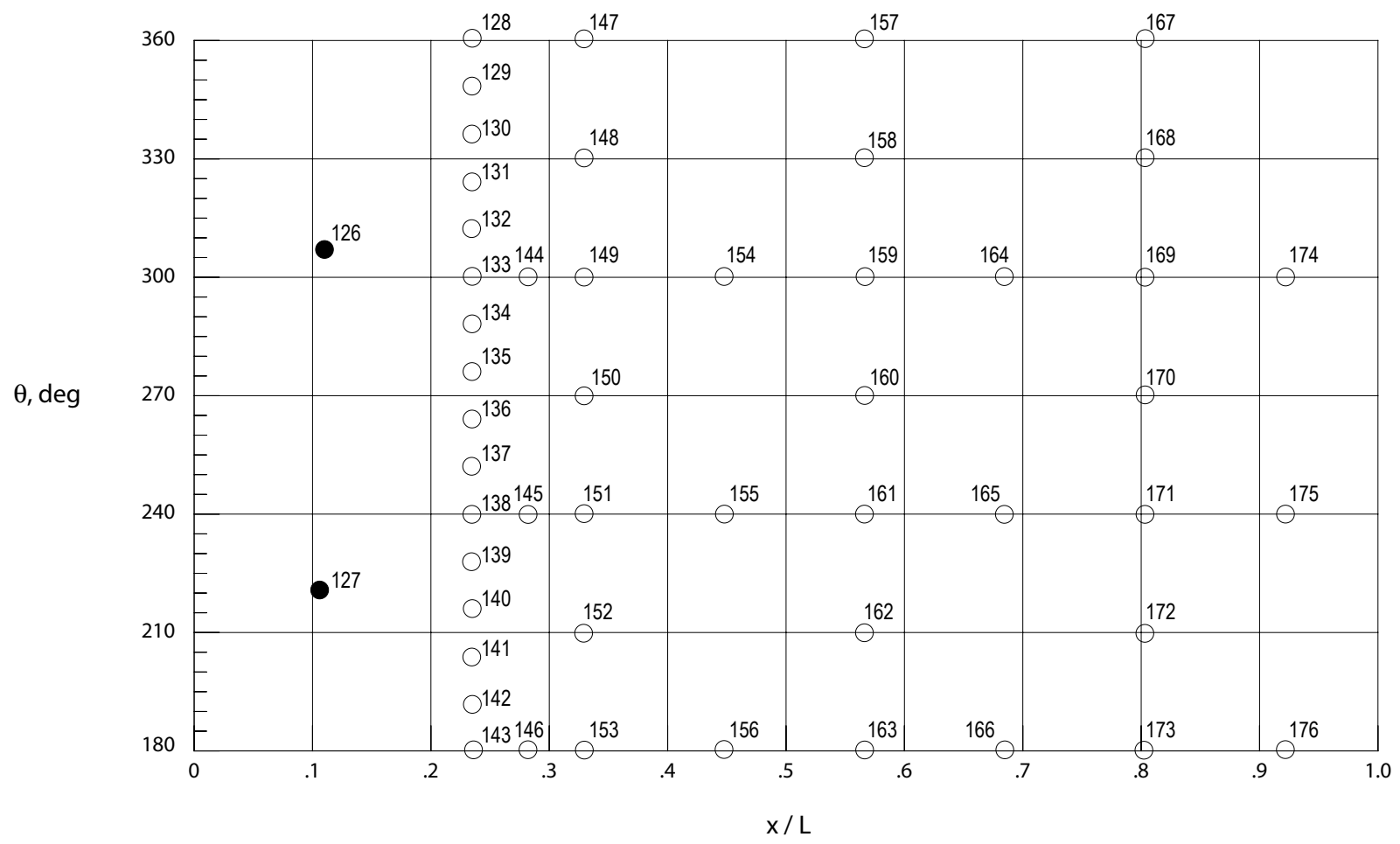

(b) Aftbody shell as viewed from the port $(-y)$ side of the model.

Figure 2: Model surface instrumentation layout. The small, numbered open circles ( 0 ) are pressure taps while the small, numbered filled circles $(\bullet)$ are $40 \mathrm{kHz}$ pressure transducers. The circular outlines in (a) are the locations of the nozzle inserts, which provide options for zero to four jets. 


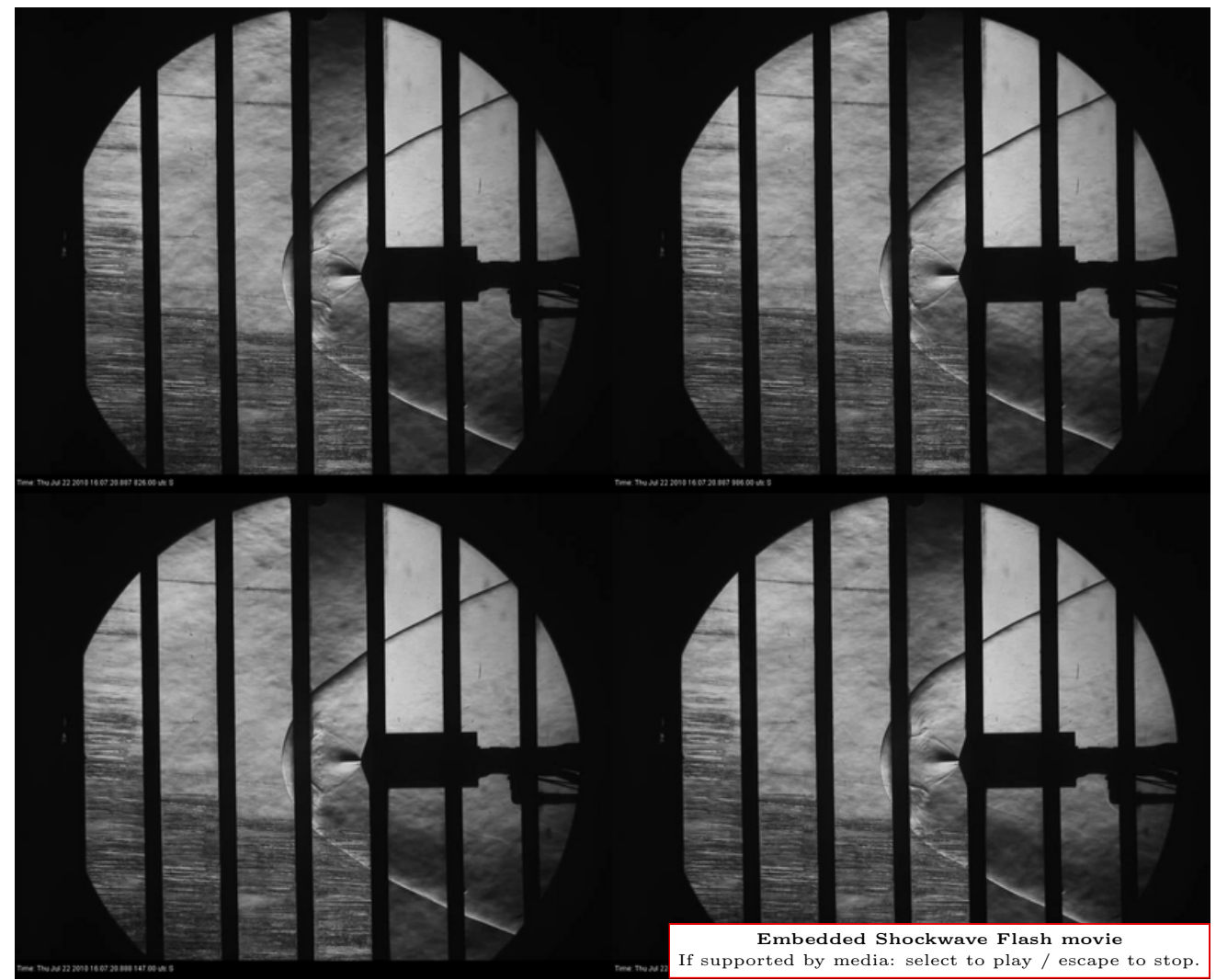

(a) $\alpha=0^{\circ}$

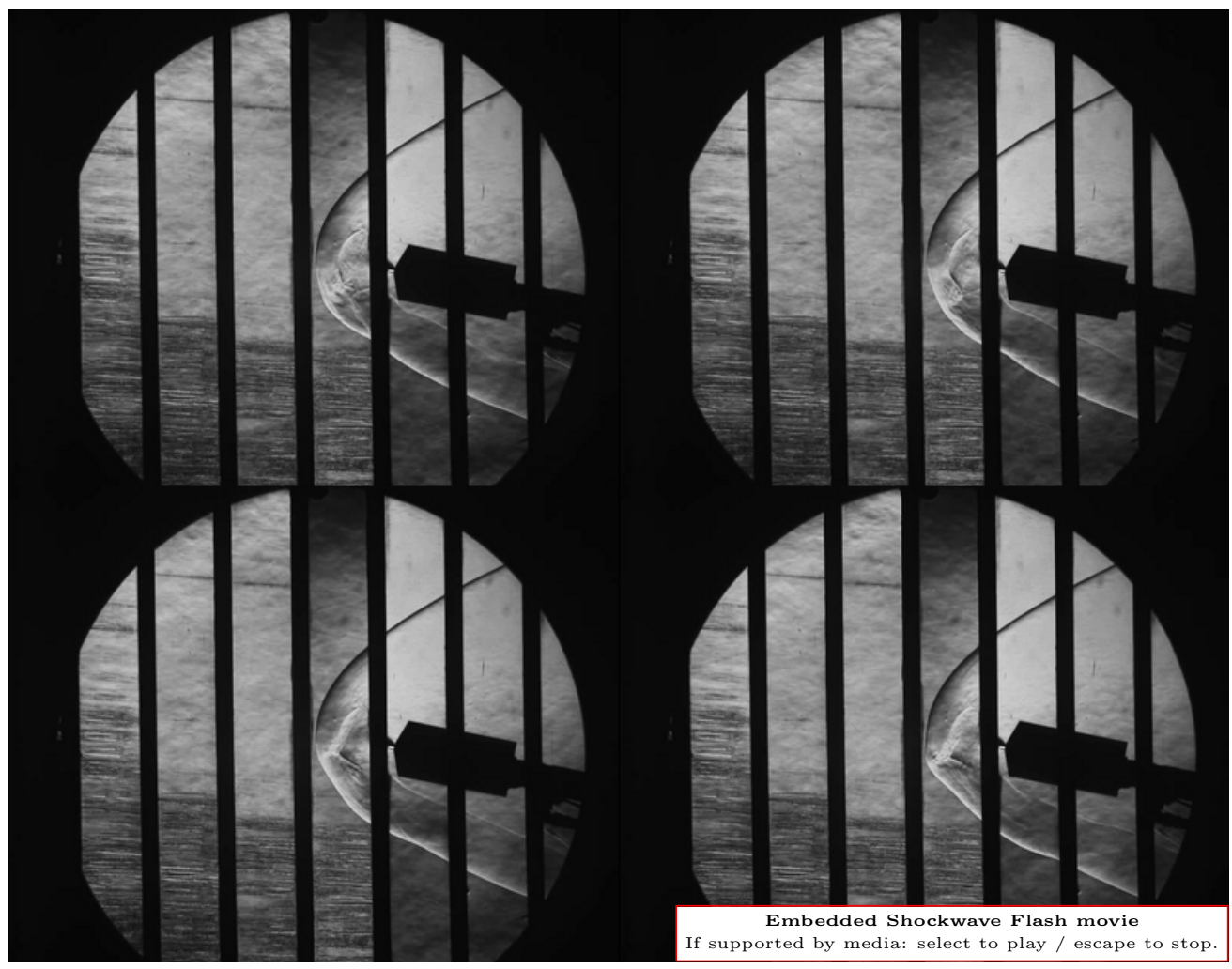

(b) $\alpha=12^{\circ}$.

Figure 3: Schlieren movies for Run 165 from Ref. 17. The static image shows a tableau of four representative still frames. 


\section{Numerical Methods, Sensitivities, and Verification}

Four types of Reynolds-Averaged Navier-Stokes (RANS) CFD codes were used to simulate SRP flows: cell-centered overset structured grid (DPLR), node-centered unstructured grid (FUN3D), node-centered overset structured grid (OvERFLOW), and cell-centered unstructured grid (Us3D). All codes simulated timedependent, fully-turbulent flow. The following subsections contain a brief summary of each code, how each was run, and a description of code and solution verification ${ }^{6,25}$ studies performed to date.

\section{A. DPLR}

The DPLR (Data Parallel Line Relaxation) CFD code ${ }^{19}$ is a parallel, structured multi-block, finite-volume code with overset grid capability that solves the RANS equations for continuum flow, including finite-rate chemistry and thermal non-equilibrium. In the present study, the thermally- and calorically-perfect RANS equations for air are solved implicitly with 1st-order time accuracy. Inviscid fluxes are formed via a modified Steger-Warming flux vector splitting ${ }^{26}$ with 3 rd-order Monotone Upwind Scheme for Conservation Laws (MUSCL) extrapolation ${ }^{27}$ subject to a minmod limiter ${ }^{28}$ and 2nd-order trapezoidal rule flux integration. The viscous fluxes are computed with 2nd-order spatial accuracy with a central difference approach. For the present analysis, the Menter's ShearStress Transport (SST) turbulence model was employed with a vorticity-based production term ${ }^{29}$ and no compressibility corrections.

A design-order verification study was conducted for an inviscid, axisymmetric nozzle problem described by Roy. ${ }^{30}$ This problem is smooth in the sense that it does not contain shocks, which reduce the scheme's design order of accuracy per Godunov's theorem. Figure 4 shows a plot of L2 error versus grid refinement factor, $h$, for 2nd-order and 3rd-order MUSCL flux reconstructions. Both options demonstrate nominally 2nd-order convergence for this smooth flow with 1.9 and 1.7 slopes, respectively. The 3rd-order scheme has slightly lower overall error but begins to degrade as the mesh is refined.

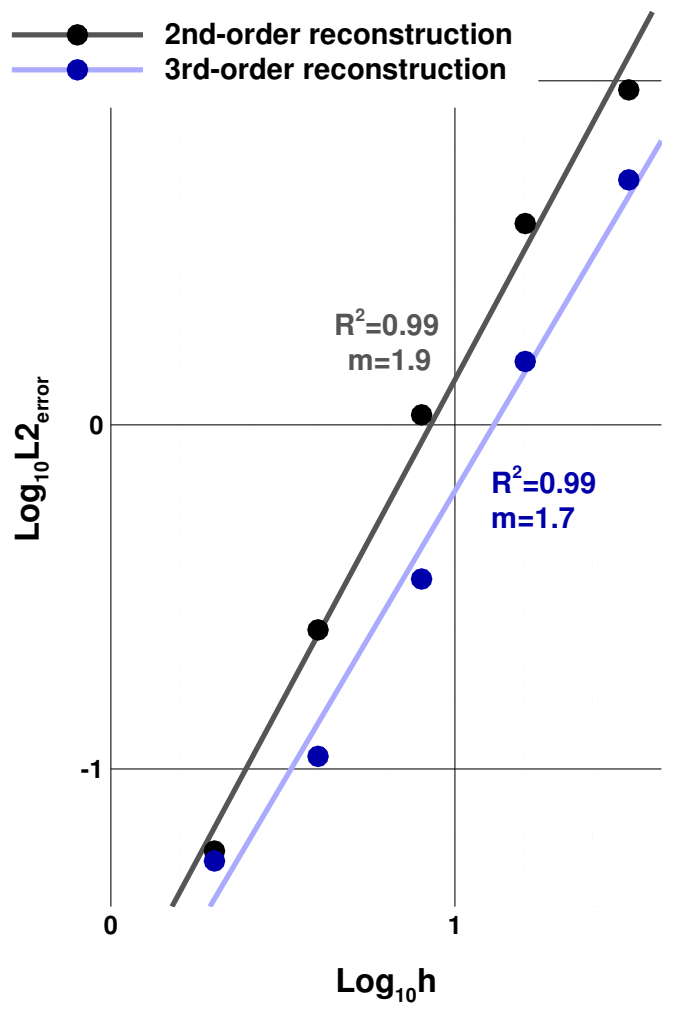

Figure 4: DPLR observed order-of-accuracy study for axisymmetric nozzle flow. 3rd-order reconstruction was used for subsequent results shown in this paper.

A topology and grid sensitivity study was performed for Run $165, \alpha=0^{\circ}$ conditions with three grids: Grid 1 with 84M nodes, Grid 2 with 18M nodes, and Grid 3 with 53M nodes. Grids 1 and 2 differ with respect to the topology of the overset regions in the jet interaction region while Grid 3 is a uniform refinement of Grid 2. Grid 2 provided better resolution of the plume region than Grid 1 with only $20 \%$ of the points. Based on comparison of the two solutions, this adjustment demonstrated that points far from areas of interest were not influential.

Figure 5 shows a comparison of predicted surface pressures by all three grids along the model centerline $\left(\theta=0^{\circ}, 180^{\circ}\right)$. As the overset topology is changed and the grid is refined, the solutions show improvement with respect to the amount of asymmetry predicted for this symmetric flow as indicated by the decreasing spread between the upper and lower centerline values. Grid 3 was considered the best compromise and was used for the predictions in the Preliminary Comparisons section on page 20. 


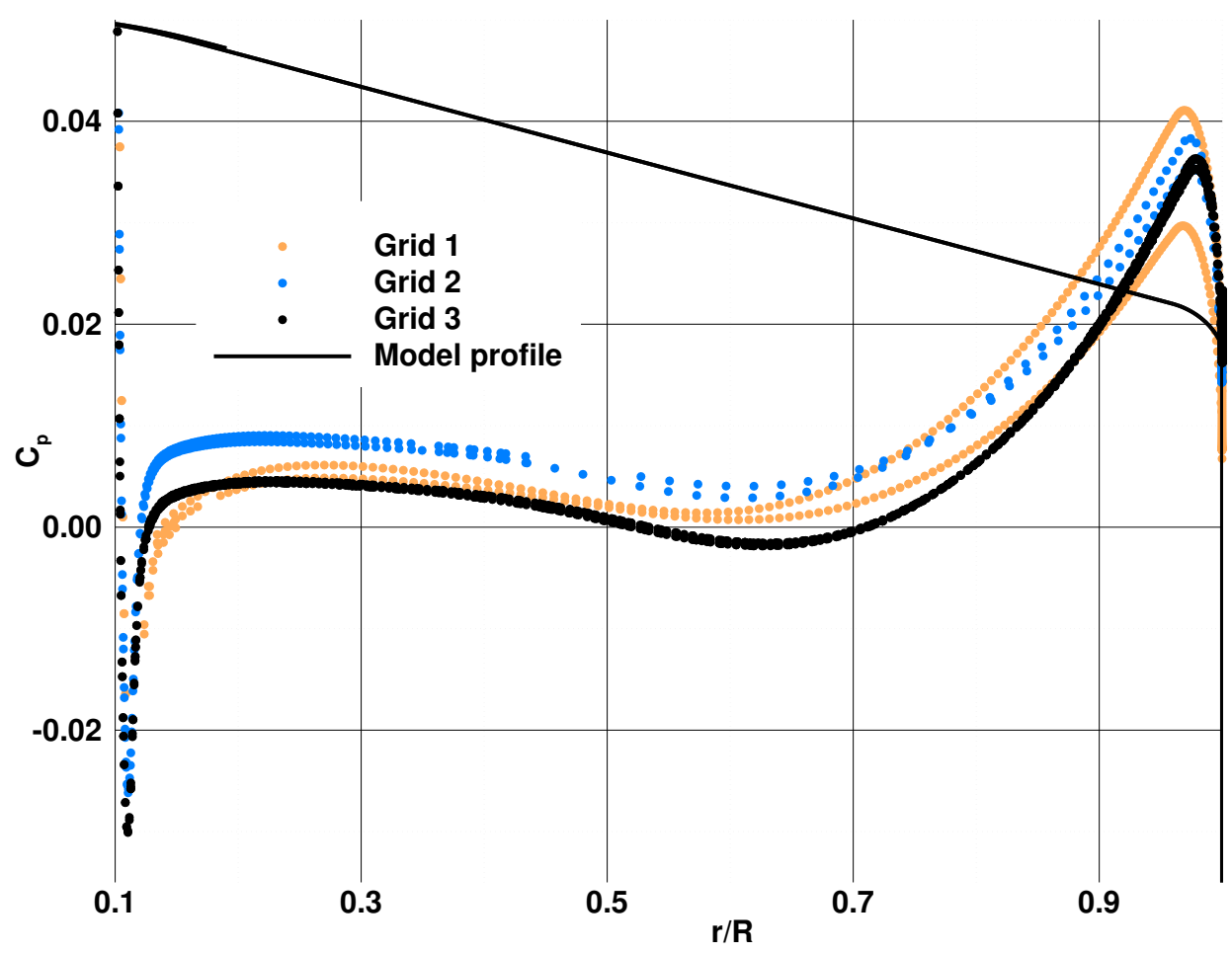

(a) Forebody only.

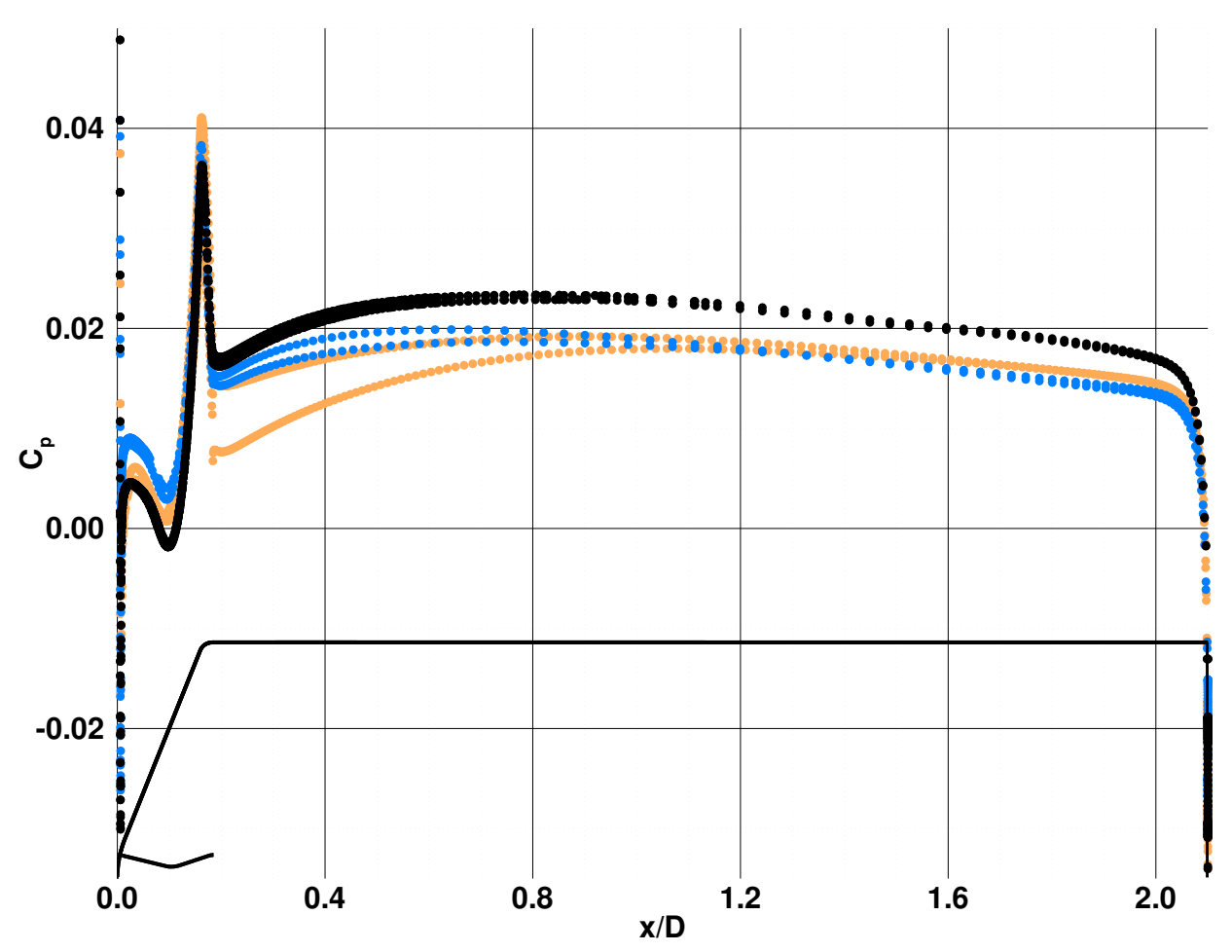

(b) Entire body.

Figure 5: DPLR grid resolution and topology study. 
Results from a time step resolution study are shown in Figures 6 and 7 where Figure 6 is a detailed view of first portion of Figure 7. Both figures show simulated pressure traces of two forebody tap locations, ${ }^{\mathrm{a}} 105$ and 106, for a period of time with up to four different time steps: the nominal $0.32 \mu \mathrm{s}$, one ten times larger, another ten times smaller, and in the case of Figure 6, another a hundred times smaller. As shown by both figures, the time step ten times larger than the nominal time step shows immediate and significant departure from all others while the difference between two smallest time steps, a tenth and a hundredth as small as the nominal (available only in Figure 6 ) is imperceptible on these expanded $C_{p}$-scale plots. Figure 7's five times longer time duration and much larger $C_{p}$ range that encompasses typical fluctuations shows that the difference between the nominal time step and one ten times smaller is negligible, and so the nominal time step was used for the rest of the study.

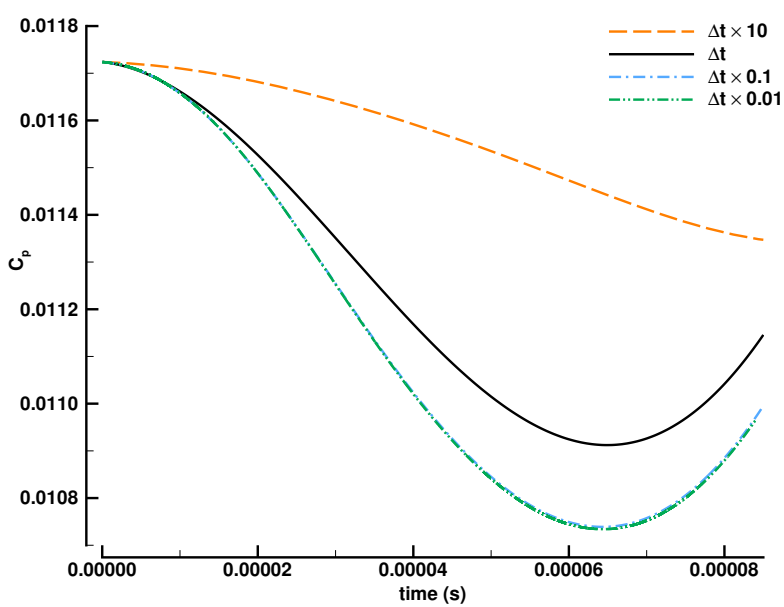

(a) Forebody tap 105.

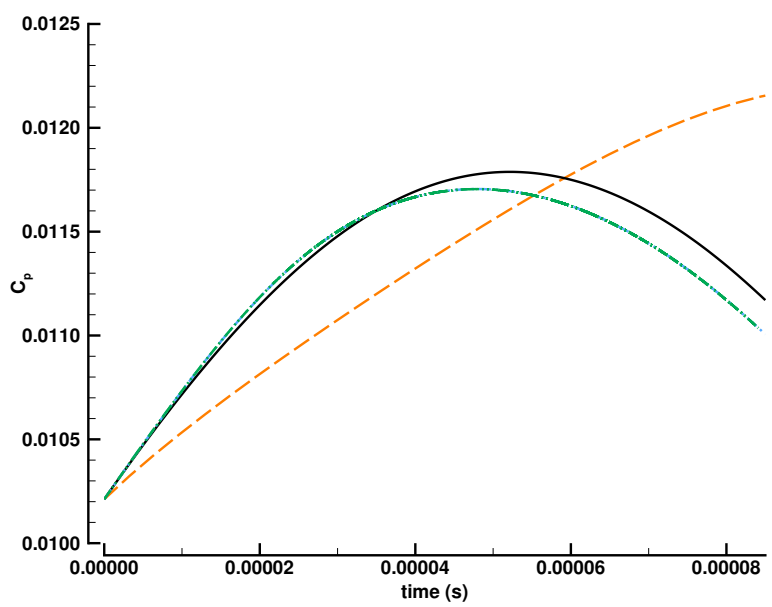

(b) Forebody tap 106.

Figure 6: DPLR time step resolution study: short duration with time steps spanning four orders of magnitude.

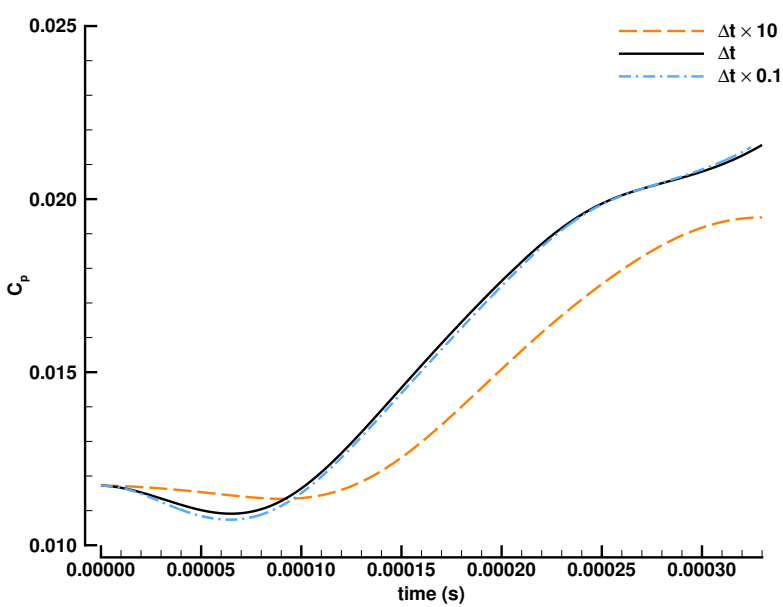

(a) Forebody tap 105.

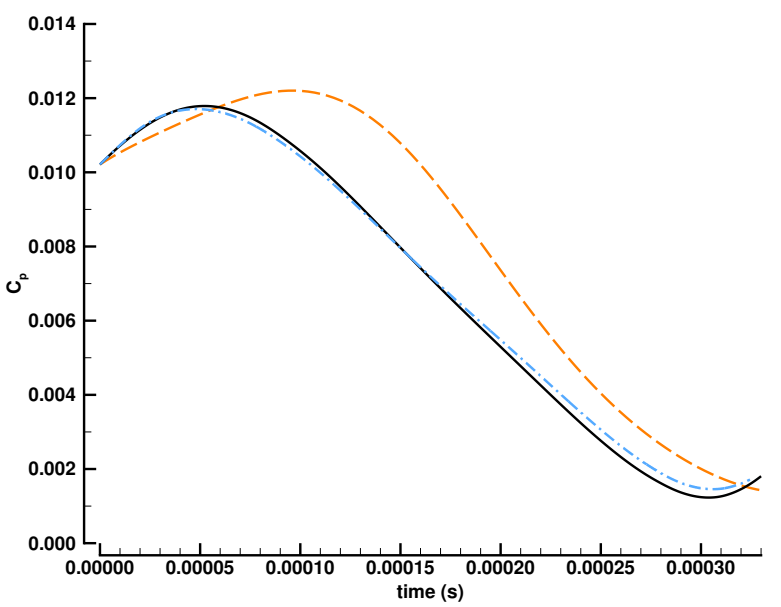

(b) Forebody tap 106 .

Figure 7: DPLR time step resolution study: longer duration with time steps spanning three orders of magnitude.

\footnotetext{
${ }^{a}$ In the full study, ten pressure tap locations were examined and all showed behavior similar to those shown here.
} 


\section{B. FUN3D}

The Fun3D (Fully Unstructured Navier-Stokes Three-Dimensional) suite of codes contains a node-based finite-volume flow solver. ${ }^{20}$ For this study, Fun3D was run with a selectively-dissipative version of the LowDissipation Flux Splitting Scheme (LDFSS) inviscid flux function ${ }^{31}$ and a modified Van Albada limiter ${ }^{32}$ according to Vatsa and White. ${ }^{33}$ The compressible RANS equations are loosely coupled to Menter's SST turbulence model with a vorticity-based production term ${ }^{29}$ or a modified Delayed Detached Eddy Simulation model that uses the Spalart-Allmaras (SA) near-wall model, ${ }^{34}$ which will subsequently be referred to as SADES. Node-based conservative variables are computed by driving a 2nd-order accurate spatial residual to steady-state with a point-implicit iterative method. A modified, optimum 2nd-order backward difference formula (BDF) scheme is used in conjunction with a temporal error controller that assures design order. ${ }^{35}$

In addition to previous code verification studies and their associated automatic regression test suites ${ }^{36}$ solution verification in the form of observed spatial order of accuracy was attempted by monitoring two error quantities as a function of grid resolution for unsteady Run 165, $\alpha=0^{\circ}$ conditions. All grids are of the same family in that the spacing field used by the grid generator was uniformly refined from coarse to fine grids. The viscous spacing and stretching rates normal to the wall, however, were held constant. This allows more surface grid resolution as the grids are refined, but the wall-normal distribution remains fixed. Table 1 provides the range of grid sizes considered for this study. Also tabulated are the two error measures used to investigate the spatial order behavior. The first, coefficient of lift $\left(C_{L}\right)$ should be zero for this zero angle of attack case and the second is a derived error by computing the difference between the coefficient of drag $\left(C_{D}\right)$ on the

Table 1: Fun3D spatial order study: number of mesh points and observed errors.

\begin{tabular}{lcc}
\hline Nodes & $C_{L}$ & $C_{D}-C_{D, \text { finest }}$ \\
\hline $3.8 \mathrm{M}$ & $2.6 \times 10^{-3}$ & $8.78 \times 10^{-4}$ \\
$6.7 \mathrm{M}$ & $1.4 \times 10^{-3}$ & $6.52 \times 10^{-4}$ \\
$10.8 \mathrm{M}$ & $7.1 \times 10^{-4}$ & $8.72 \times 10^{-5}$ \\
$19.7 \mathrm{M}$ & $1.6 \times 10^{-4}$ & $1.17 \times 10^{-4}$ \\
$28.4 \mathrm{M}$ & $3.6 \times 10^{-4}$ & $8.65 \times 10^{-5}$ \\
$43.4 \mathrm{M}$ & $4.8 \times 10^{-4}$ & $8.93 \times 10^{-5}$ \\
$72.6 \mathrm{M}$ & $1.8 \times 10^{-5}$ & - \\
\hline
\end{tabular}

finest and the current grid. For this unsteady problem, both of these measures are inherently noisy due to the time-averaging necessary to determine these quantities.

These two errors are plotted as a function of the number of nodes to the two-thirds power, $N^{-2 / 3}$, in Figure 8. On these plots, a 2nd-order scheme (the smooth-flow design order for Fun3D) has a slope of one. ${ }^{b}$ As anticipated, both subfigures show very noisy results for this non-smooth flow with such a loose family of grids. The lift error behavior is especially bad for finer grids. The non-monotonic slopes may be due to different feature sets becoming resolved as the grid is refined as shown by the simulated Schlierens in Figure 9 or simply that this complex, unsteady flow is beyond such crude measurement techniques. Perhaps running even finer grid levels would more clearly indicate a transition to 1st-order, which is hinted at in subfigure $8 \mathrm{~b}$ and is expected for non-smooth flow as the grid is refined. ${ }^{30}$

During this investigation, a simulated Schlieren capability ${ }^{\mathrm{c}}$ was added to Fun3D and provided a revealing visual depiction of grid resolution effects - see Figure 9, which shows simulated Schlieren images for three of the grids used in the spatial order study. As the grid is refined, shocks and shear layers sharpen. Due to lack of resources, however, only the medium-resolution grid (28M nodes) was used for the final simulations.

For Fun3D runs, the solution was advanced in time with an optimal 2nd-order modified BDF scheme ${ }^{35}$ with a non-dimensional time step of 0.003 at a CFL (Courant-Friedrichs-Lewy) number of five. This time step, which gave approximately 200 steps per cycle, corresponds to a physical time step of $2.36 \mu$ s. A maximum of 20 sub-iterations were used for each time step. A temporal error controller was used to assure temporal accuracy. If the $x$-momentum and turbulent kinetic energy L2 error norms dropped an order of magnitude below the temporal error estimate, the solution was advanced to the next time step.

\footnotetext{
${ }^{\text {b The }} N^{-2 / 3}$ combines a measure of the mesh spacing, $h \sim N^{-1 / 3}$, and 2nd-order behavior with respect to spacing, $h^{2}$.

${ }^{\mathrm{c}}$ Simulated Schlieren images are generated via volume integration as described by Yates. ${ }^{37}$
} 


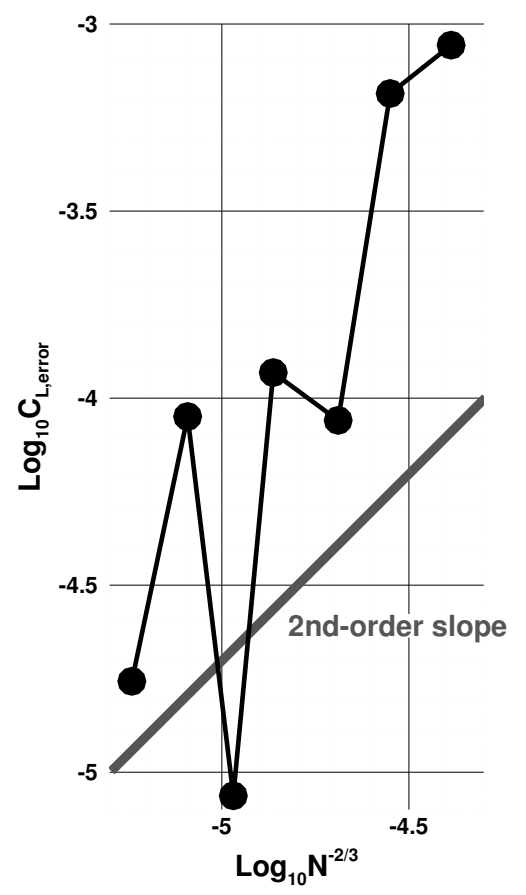

(a) $C_{L}$.

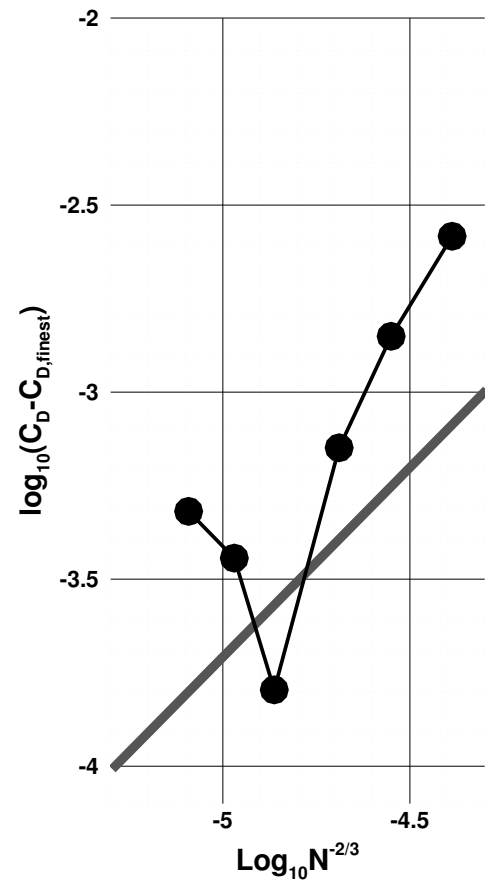

(b) $C_{D}-C_{D, \text { finest }}$.

Figure 8: FUN3D observed spatial order behavior. On this plot, unity slope represents 2nd-order convergence.

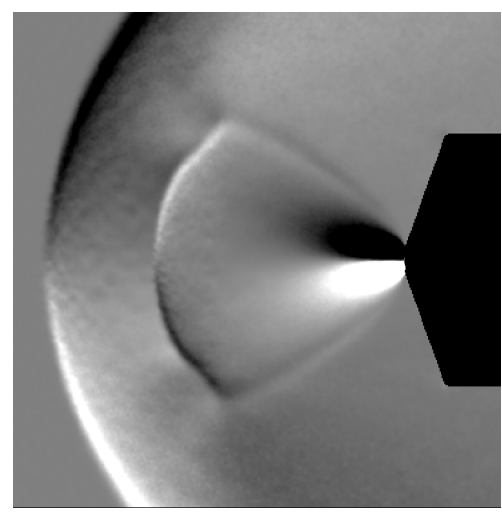

(a) $3.8 \mathrm{M}$ nodes.

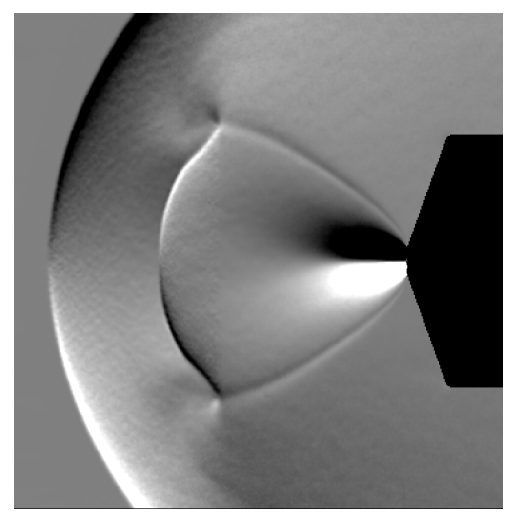

(b) $28.4 \mathrm{M}$ nodes.

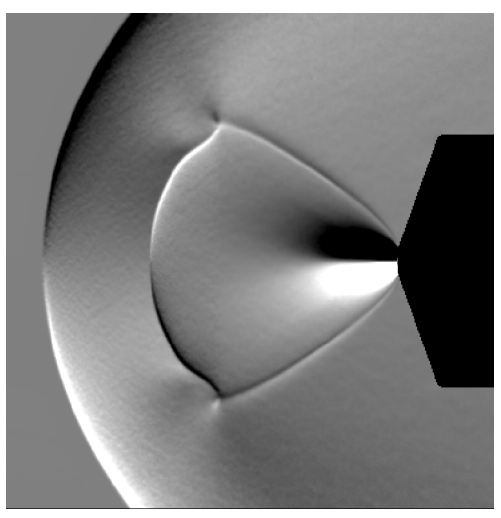

(c) $72.6 \mathrm{M}$ nodes.

Figure 9: Visualizing grid resolution effects via $700 \times 700-$ pixel simulated Schlieren (FUN3D). 


\section{OVERFLOW}

OvERFLOW 2 (OVERset grid FLOW solver) is an implicit RANS flow solver that utilizes structured overset grids. ${ }^{22}$ For the current work, the HLLE++ numerical flux function ${ }^{38}$ with the Van Albada limiter ${ }^{32}$ was used for spatial terms, and the Symmetric Successive Over Relaxation (SSOR) algorithm with Newton subiterations for temporal terms. All viscous terms were included, and turbulence was modeled with either Menter's original SST model $^{39}$ subject to Wilcox's realizability constraint ${ }^{40}$ or a SSTDES hybrid model. ${ }^{41}$ The overall scheme is 2nd-order accurate Table 2: Overflow axisymmetric grid sensitivity study.

\begin{tabular}{lrc}
\hline Name & Nodes & Refinement/dir \\
\hline coarse & 169,710 & 1.00 \\
medium & 641,127 & 1.94 \\
fine & $2,656,902$ & 3.96 \\
\hline
\end{tabular}
in space and time. The calculation of inviscid fluxes for both the flow solver and the turbulence model use 3rd-order accurate MUSCL reconstruction.

A grid sensitivity study was conducted with the three axisymmetric grids described in Table 2. The grid spacing levels are based on a global refinement factor where as the refinement factor decreases, refinement increases. A couple views of the finest grid are shown in Figure 10. Run $165, \alpha=0^{\circ}$ conditions were used and the domain was modeled without tunnel walls.

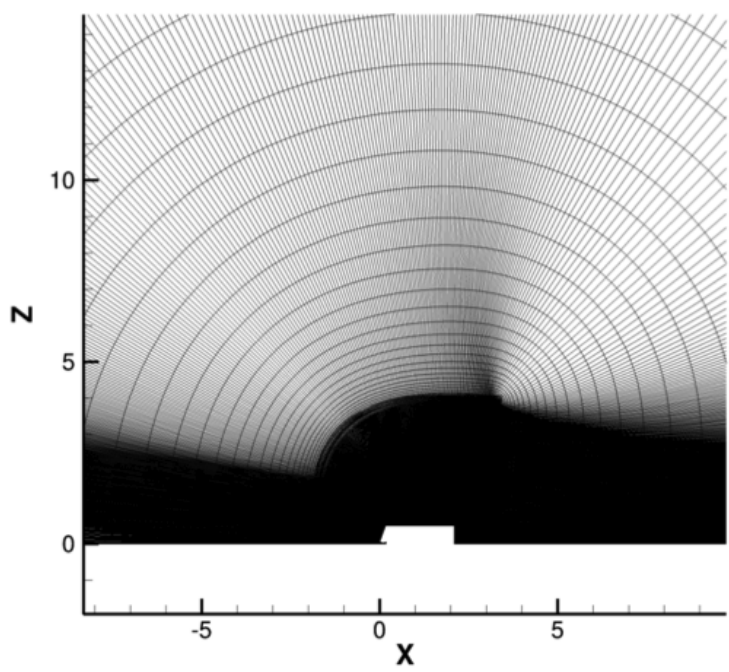

(a) Midrange view.

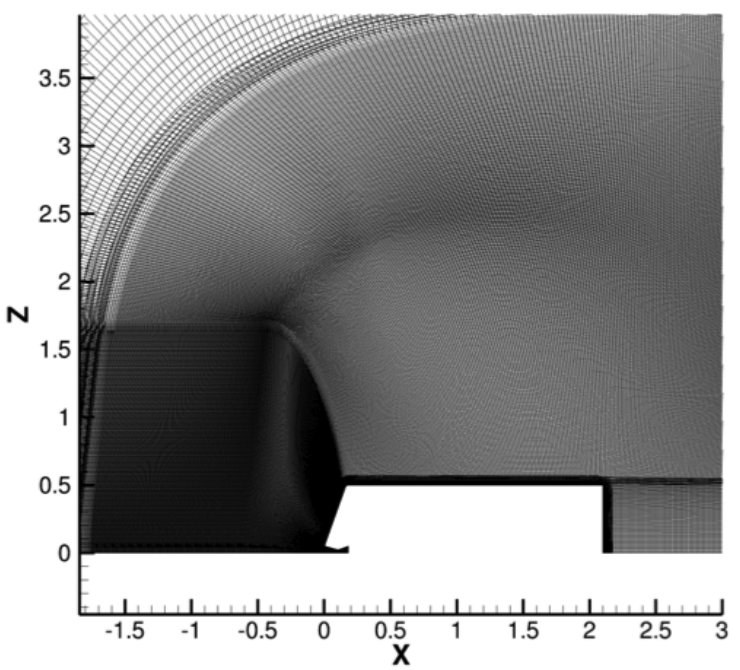

(b) Close up.

Figure 10: Fine grid used for OVERFLOW axisymmetric grid convergence study.

Figure 11 shows time-averaged solution comparisons for three grid resolutions. For $C_{p}$ on the model face, the fine and medium grids are similar while the coarse grid is low. Downstream of the model shoulder shows a lot of difference in $C_{p}$ between refinement levels. Meanwhile, the shock standoff distances of all refinements are similar with the coarse grid being slightly farther forward.

To gauge the differences in unsteadiness captured by the three grid resolutions, the frequency of the drag force oscillations were computed as 1.7, 2.0 , and $2.3 \mathrm{kHz}$ from coarse to find grids, respectively. The fine and medium grids are similar in oscillation period and drag amplitude while the coarse grid has both smaller period and amplitude. The higher the refinement, the more detail captured; and similarly, the amount of unsteadiness increases with grid refinement. For the fine and medium grids, the average $C_{p}$ on frontal face is very similar and the average $C_{p}$ on side body oscillate near each other. Shedding from the tripoint region increases average $C_{p}$ on model face; and for time-averaged results, the medium grid yields answers comparable to the fine grid at a fraction of the cost.

Another study was conducted to measure spatial order of accuracy. Here, a family of five axisymmetric grids were used as shown in Table 3. As with Fun3D, the error in $C_{D}$ with respect to the finest mesh was used to monitor convergence. Figure 12 shows the error as a function of mesh spacing. A linear fit of the points gives a slope of 1.0, indicating 2nd-order spatial accuracy. 


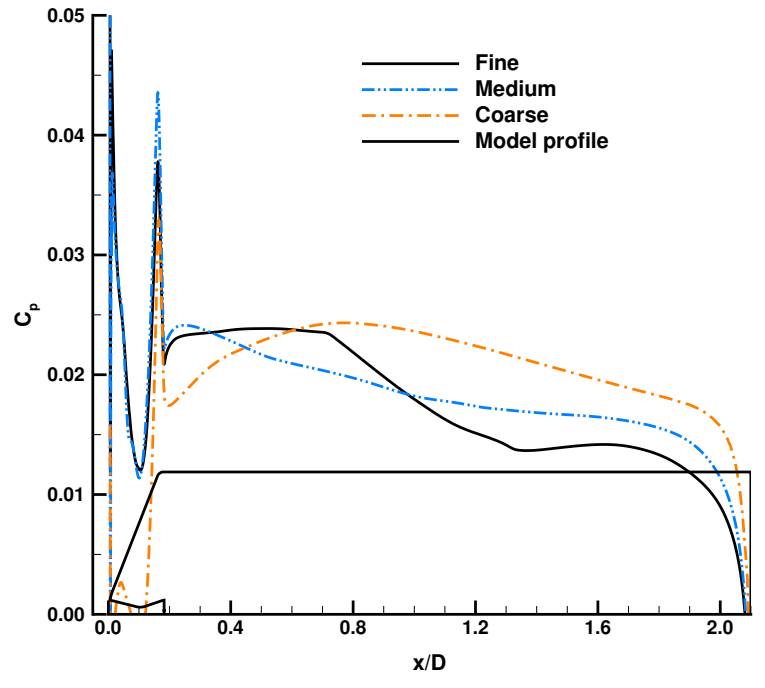

(a) $C_{p}$ vs $x / D$.

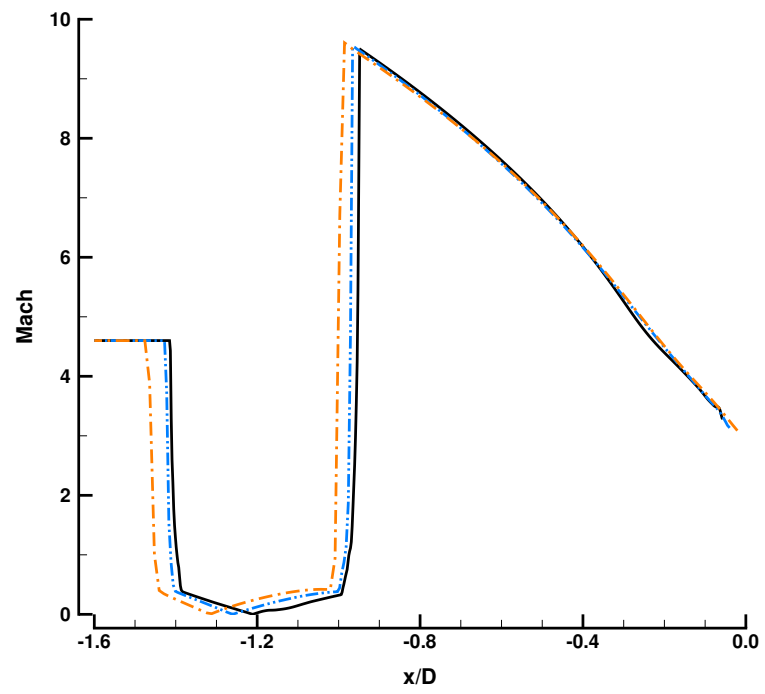

(b) Centerline Mach vs $x / D$.

Figure 11: OVERFLOW time-averaged solution comparison for three grid resolutions.

Table 3: OvERFLOW axisymmetric grids for spatial order study.

\begin{tabular}{lrc}
\hline Name & Nodes & Refinement/dir \\
\hline Grid 1 & 169,710 & 1.00 \\
Grid 2 & 287,046 & 1.30 \\
Grid 3 & 641,127 & 1.94 \\
Grid 4 & $1,098,819$ & 2.54 \\
Grid 5 & $2,656,902$ & 3.96 \\
\hline
\end{tabular}

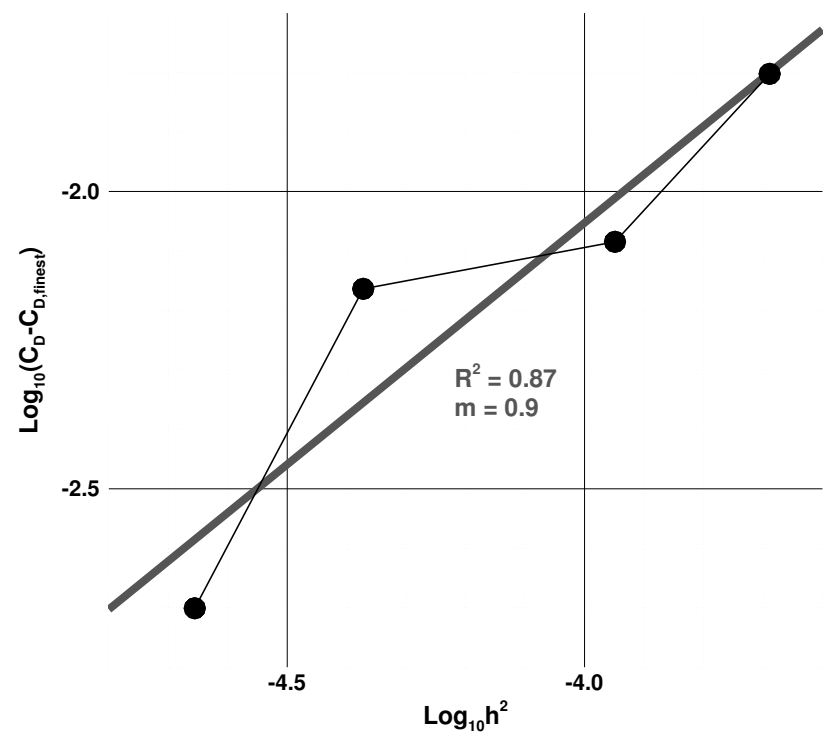

Figure 12: OvERFLOW observed spatial order of accuracy for axisymmetric simulation of Run $165, \alpha=0^{\circ}$. 
Next, the number of subiterations necessary during time advancement was investigated. Starting from time step 11,500 on the medium grid with a $1.71 \mu \mathrm{s}$ time step, 5,10 , and 20 subiterations were run. As expected, the drop in subiteration L2 residuals increases with number of subiterations. No variation in drag or frequency was seen, and therefore five subiterations were used henceforth.

Finally a time step sensitivity study was performed where the time step was varied though three orders of magnitude: 17.1, 1.71, and $0.171 \mu \mathrm{s}$. Starting at the same point in time, each was run for $2,760 \mu \mathrm{s}$ as shown in Figure 13. The largest time step, $17.1 \mu \mathrm{s}$, has lower frequency than the smaller time steps, For time steps of $1.71 \mu \mathrm{s}$ and $0.171 \mu \mathrm{s}$, drag behavior is very similar. Time-averaged shock standoff distances, as shown in Figure 14, are not very sensitive to time step. The two smallest time steps are nearly identical in time-averaged $C_{p}$ distributions. Drag oscillation frequencies as a function of decreasing time step size were $1.17,1.83$, and $1.99 \mathrm{kHz}$, respectively. As time step is reduced, the frequency of the tripoint oscillation increases. In conclusion, to get average flow field properties, a time step of $1.71 \mu \mathrm{s}$ is sufficient.

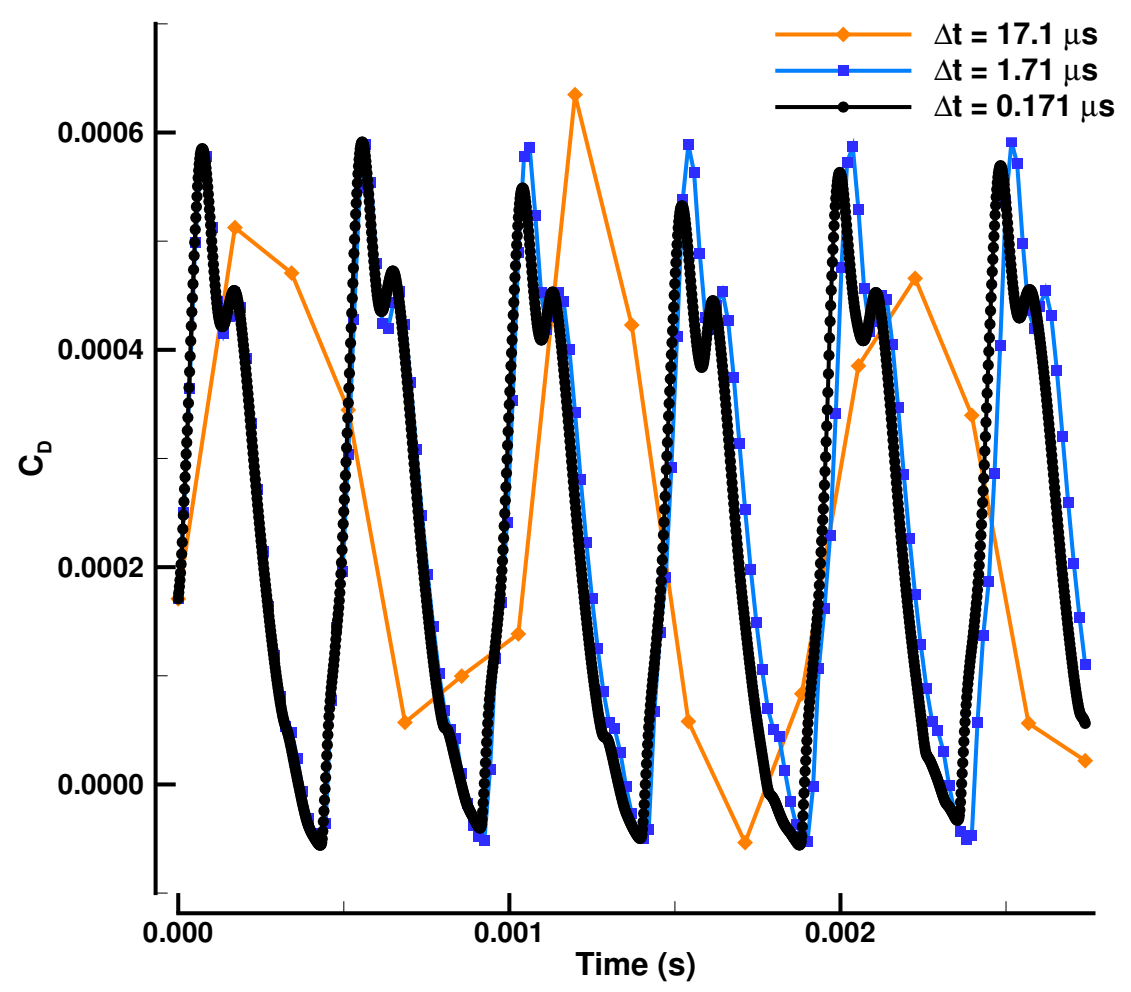

Figure 13: Overflow $C_{D}$ as a function of time step. 


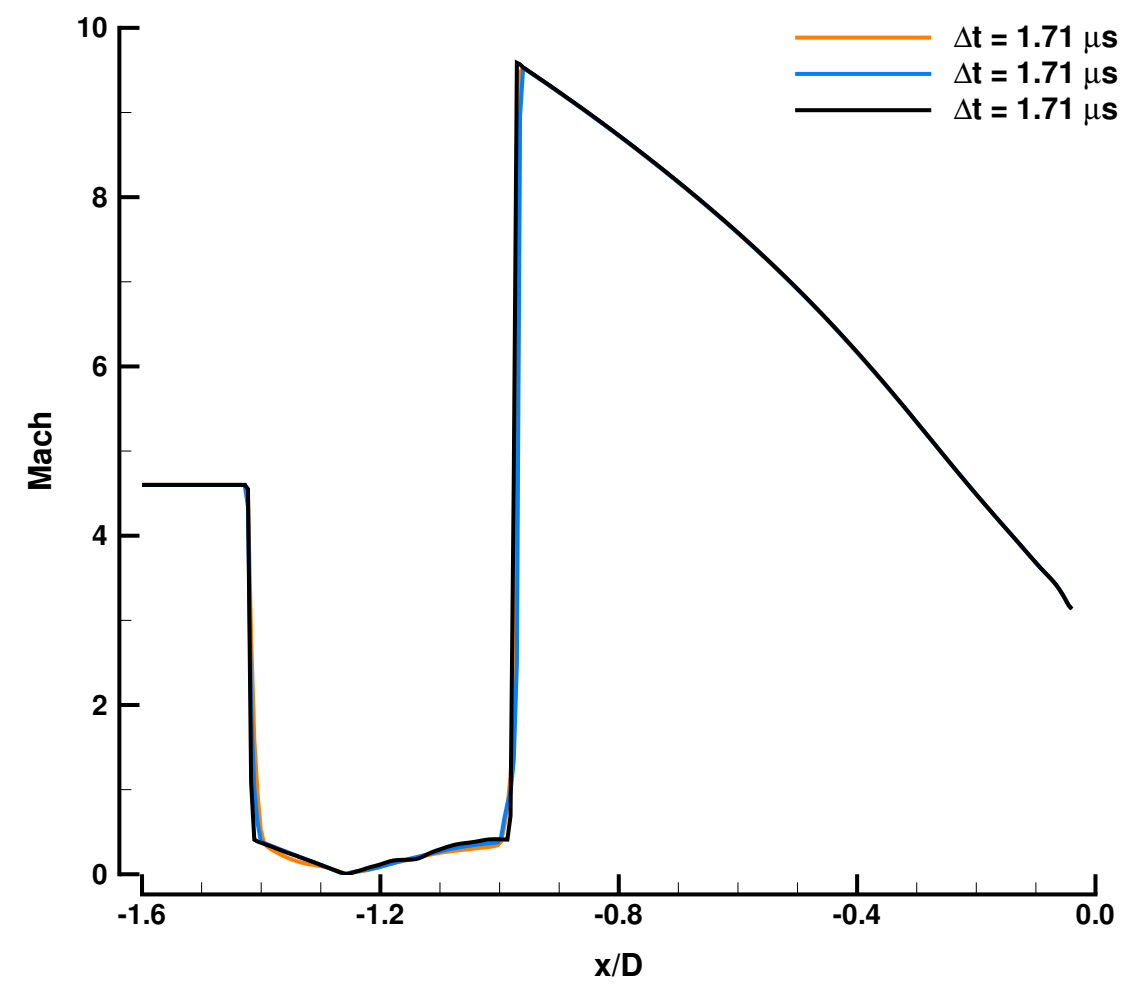

Figure 14: OvERFLOW time-averaged shock standoff distance as a function of time step.

\section{US3D}

The Us3D (Unstructured Three-Dimensional) $\operatorname{code}^{23,42}$ is a parallel, Navier-Stokes, finite-volume solver for unstructured grids. It shares roughly the same features, numerical methods and physical models as DPLR including the finite-rate chemistry and thermal non-equilibrium with a major difference being the unstructured grid capability of Us3D. For a given problem using identical grids and modeling options, the two codes produce the exact same solutions to machine precision.

Us3D is a relatively new code and is still in development phase. As of this writing, two equation turbulence models are yet to be implemented. In the current study, SA-DES turbulence modeling is used. ${ }^{43,44}$ Both inviscid and viscous fluxes are evaluated with 2nd-order spatial accuracy, and backward Euler time integration provided 1st-order temporal accuracy.

Unfortunately, Us3D grid and time resolution sensitivity studies have not yet been completed for the problem studied herein. The grid employed consisted of 31.4 million hexahedral cells, and the time step was fixed at $1.5 \mu \mathrm{s}$. 


\section{Physical Modeling Studies}

In addition to code verification and solution verification discussed above, three aspects of physical modeling were explored: wind tunnel wall interference, the effect of the model support hardware and high-pressure feedline, and turbulence modeling. These were explored to determine what level of physical modeling was necessary to capture the flow physics.

\section{A. Tunnel Wall Effects}

Figures 15 and 16 show the effects on the time-averaged surface pressures of modeling inviscid tunnel walls or not with Overflow. For these angles of attack, there are no substantial differences in surface $C_{p}$ and the differences seen in the forebody distribution of Figure $16 \mathrm{a}$ is assumed to be due to insufficient time-averaging. This $12^{\circ}$ angle of attack case contains large-scale unsteadiness of the entire flowfield, and the original practice of averaging over several thousand time steps was later found to be insufficient when monitoring root-mean-square fluctuations.

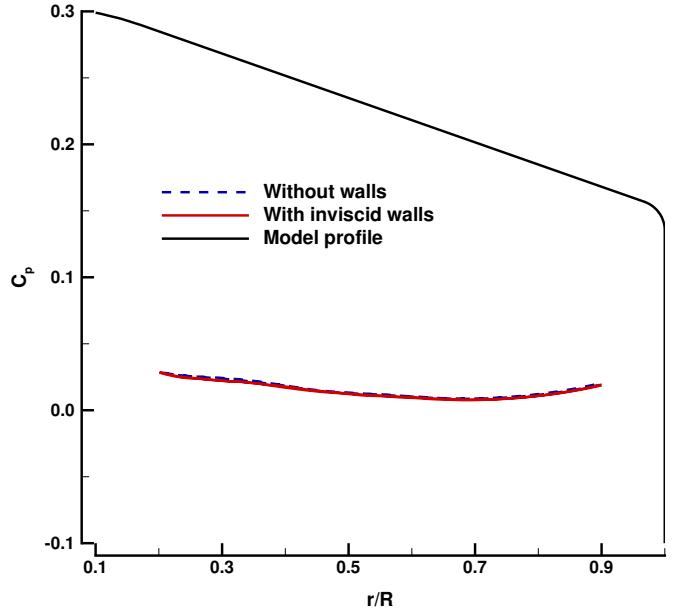

(a) Forebody only.

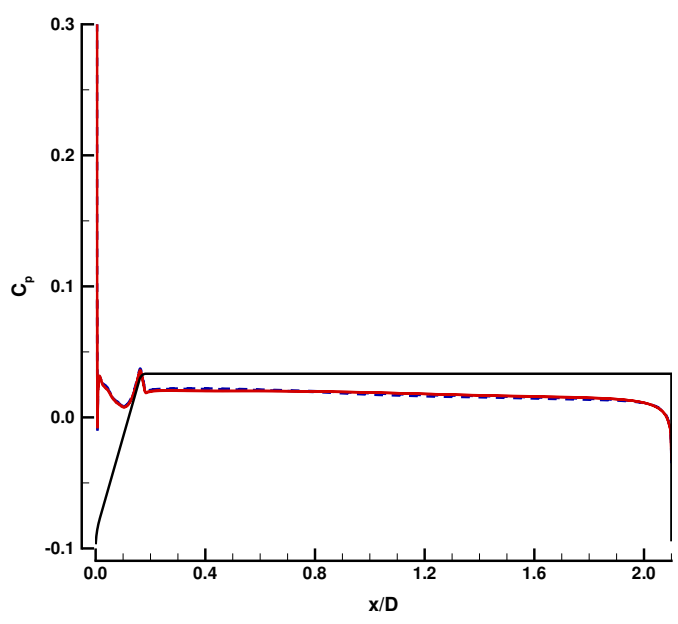

(b) Entire body.

Figure 15: Effect of inviscid tunnel walls on forebody $C_{p}$ for Run 165 at $\alpha=0^{\circ}$.

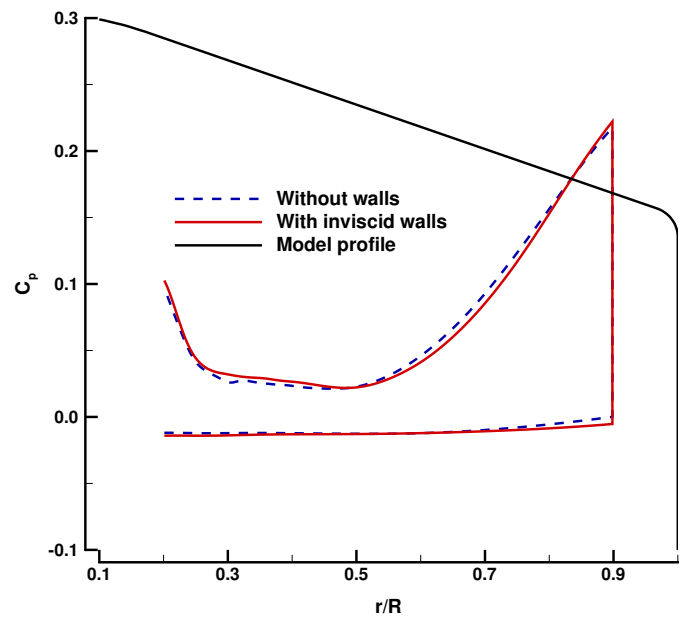

(a) Forebody only.

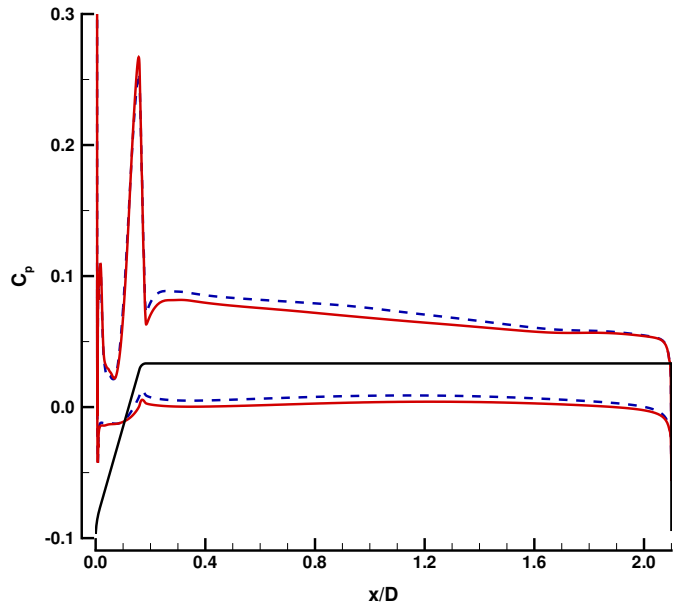

(b) Entire body.

Figure 16: Effect of inviscid tunnel walls on surface $C_{p}$ for Run 165 at $\alpha=12^{\circ}$. 


\section{B. Sting Effects}

On the sting, the high pressure feedline and instrumentation wiring bundles caused protuberances close to the model base. Since a subsonic wake region existed in this area, it was possible that influence from the protuberances traveled upstream and affected pressure tap data. To quantify any discrepancy in pressure tap readings due to the sting, an overset mesh was created from a simplified sting geometry, and cases were simulated using Overflow. The goal was to show whether including the sting in the CFD models was a requirement for good comparison to surface pressure test data.

The model was greatly simplified to get a rough idea of 1st-order effects as shown in Figure 17. Dimensions were estimated from photographs; and while not exact, they should be close enough for the purposes of this study. As seen in the figure, the sting was modeled only to a certain point downstream. Cavities under the white fiberglass tape holding the feedlines to the sting were considered solid, and the instrumentation wiring bundle was not modeled.

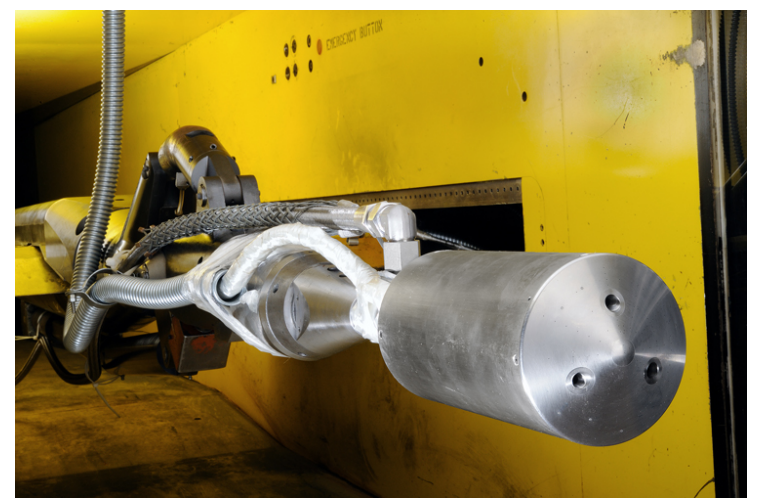

(a) Experiment. (See also Figure 1.)

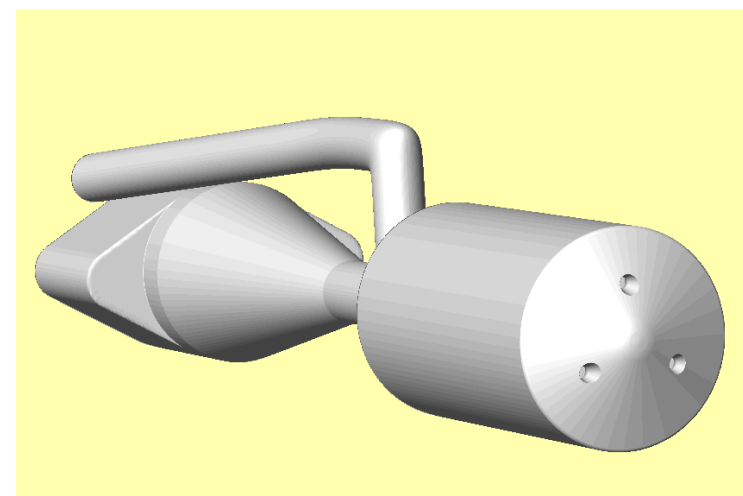

(b) Simplified model.

Figure 17: Model mount modeling.

Four cases were simulated at Mach 2.4, Re/ $f t=1 M$, and $C_{T}=3$. They were a single nozzle configuration at zero angle of attack and a triple nozzle configuration at angles of attack $-8,0$, and 12 degrees. Each case was run with and without the sting, and surface pressure comparisons were conducted. A sample flowfield with the sting is shown in Figure 18 for the tri-nozzle configuration at $\alpha=0^{\circ}$.

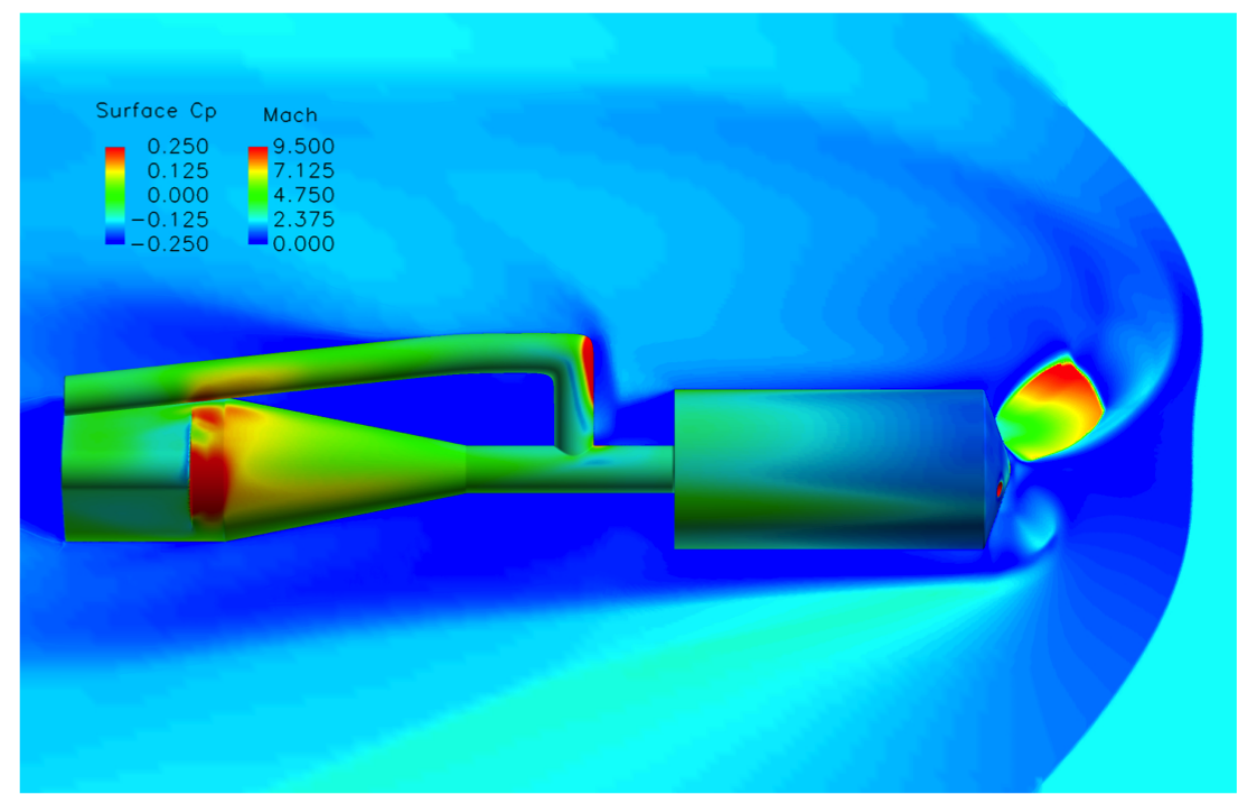

Figure 18: Sting flow. 
Comparisons of time-averaged surface pressures for the tri-nozzle configuration at zero angle of attack case are shown in Figure 19. This figure shows that the sting raised the base pressure on the model. This influence traveled upstream far enough to affect the three pressure ports that were the furthest aft on the model, taps 174-176 - see Figure 2 on page 4. The compression at the model base due to the sting also caused a decrease in drag. This study also showed changes in the unsteady fluctuations that affected the instantaneous surface pressures, but the time-averaged values were unaffected.

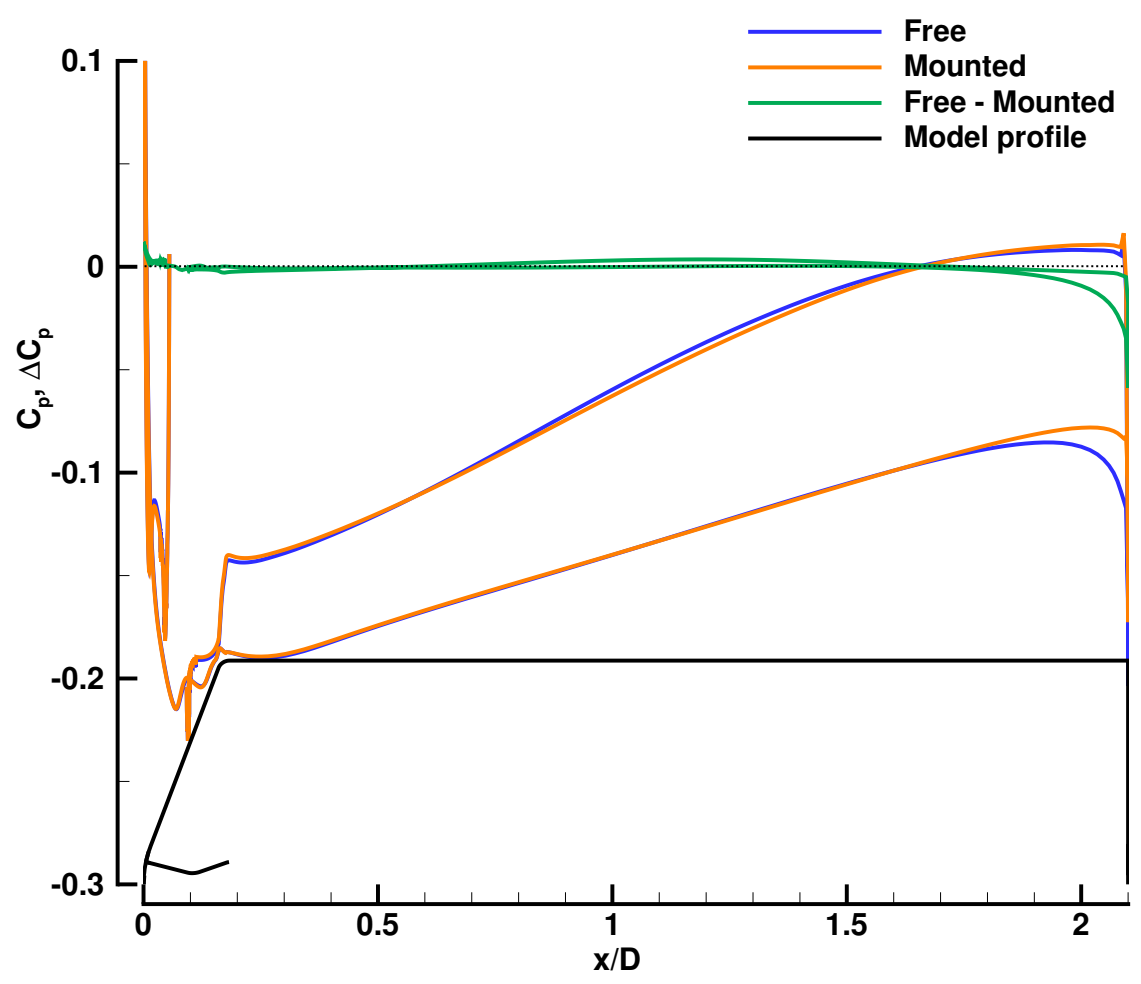

Figure 19: Sting effects.

Because the sting effects were limited to the base and only three aft pressure ports, the effort of modeling the sting had limited returns, and was deemed unnecessary for pressure comparisons over the remainder of the body. In future tests, however, where force and moment quantities are measured, the discrepancy in the model base pressure will be an important consideration because the sting causes increased base pressure and thus directly affects the model's drag. 


\section{Turbulence Modeling}

The turbulence models considered span: SpalartAllmaras (SA $)^{43}$ and two versions of Menter's $k-\omega$ Shear-Stress Transport (SST) ${ }^{29,39}$ Reynolds-Averaged Navier-Stokes (RANS) models; Spalart's Delayed Detached Eddy Simulation (DDES) model ${ }^{45}$ and Sanchez-Rocha et al's Hybrid RANS-Large Eddy Simulation (HRLES) model. ${ }^{46}$

Figure 20 shows turbulent eddy viscosity levels predicted by OvERFLOW relative to laminar (bulk) viscosity for three configurations of Menter's original SST turbulence model: (1) the baseline model, ${ }^{39}$ (2) the baseline model with a compressibility correction, ${ }^{47}$ and (3) the baseline model with Wilcox's realizability constraint. ${ }^{40}$ The turbulent eddy viscosity levels for the baseline model are high between bow and terminal shocks, which is a known, non-physical artifact. The baseline model with compressibility correction is similar in behavior, but has lower levels of eddy viscosity and the flow is more unsteady. Eddy viscosity levels for the baseline model with a realizability constraint are lower than the other models around the shocks, and higher in the wake. The realizability constraint eliminates the spurious post-shock eddy viscosity production and better matches wind tunnel plume interaction behavior.

Figure 21 shows a comparison of FUn3D timeaveraged surface pressures between the SST turbulence model and SA-DES along the upper and lower centerlines for Run 165 at zero angle of attack. In the forebody region, and especially near the jet exit at $r / R=0.1$, SA-DES recovers higher pressures than RANS. Based on solution behavior during grid convergence studies, this increase in pressure recovery is thought to be related to the extent that the jet interaction is dissipated. For the SA-DES solution, the RANS submodel is only engaged in the near-wall regions and thus does not add turbulent eddy viscosity to the jet interaction region, leading to less overall dissipation as compared to the pure RANS solution.

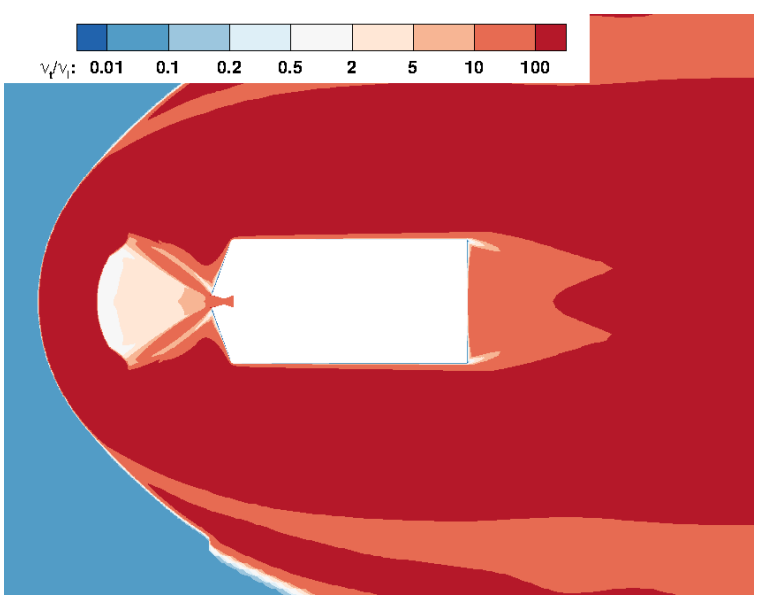

(a) Standard model.

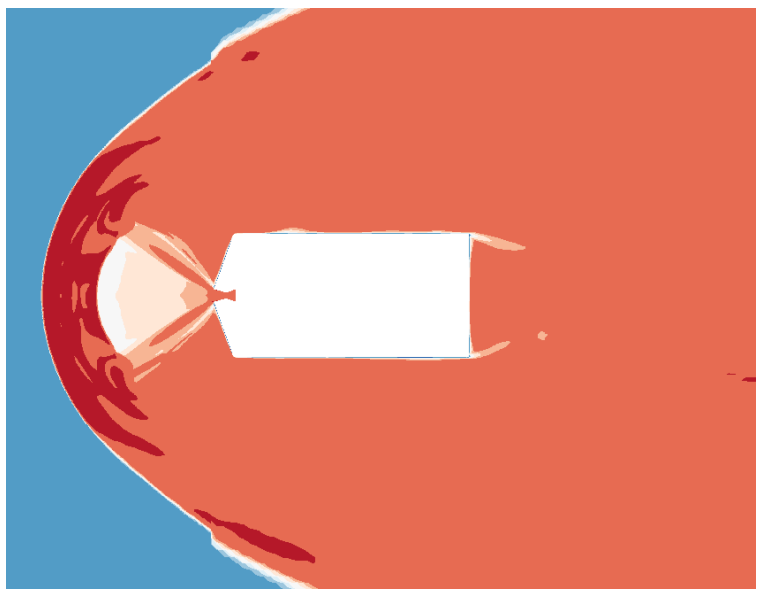

(b) Compressibility correction.

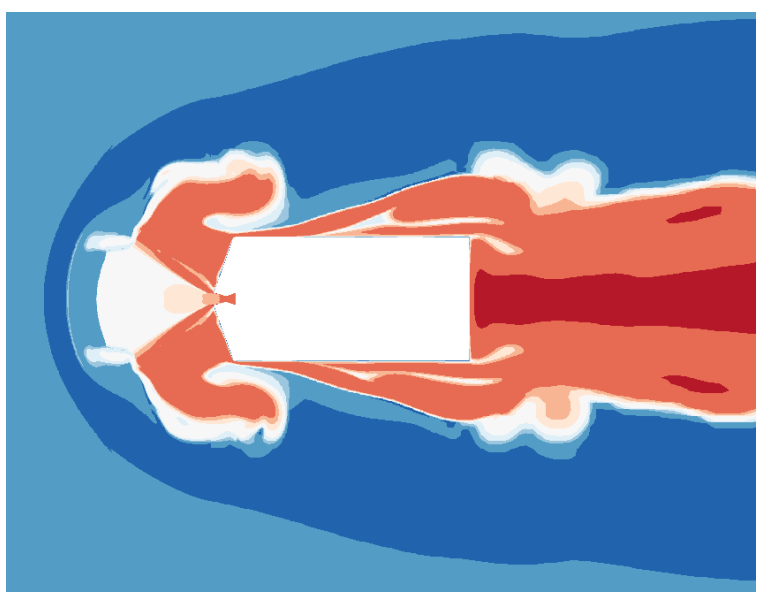

(c) Realizability constraint.

Figure 20: Relative eddy viscosity levels for various Menter SST turbulence model variations. 


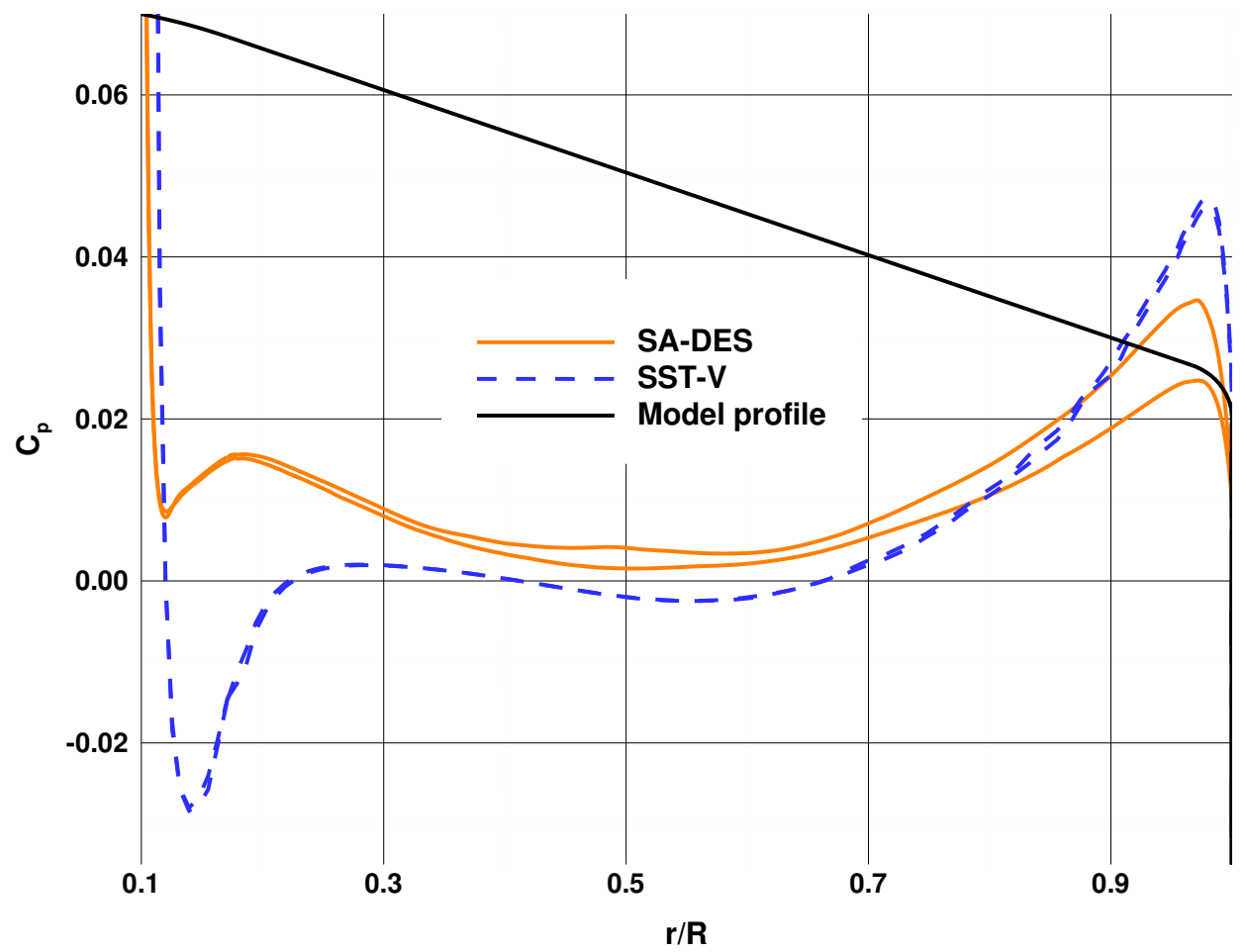

(a) Forebody only.

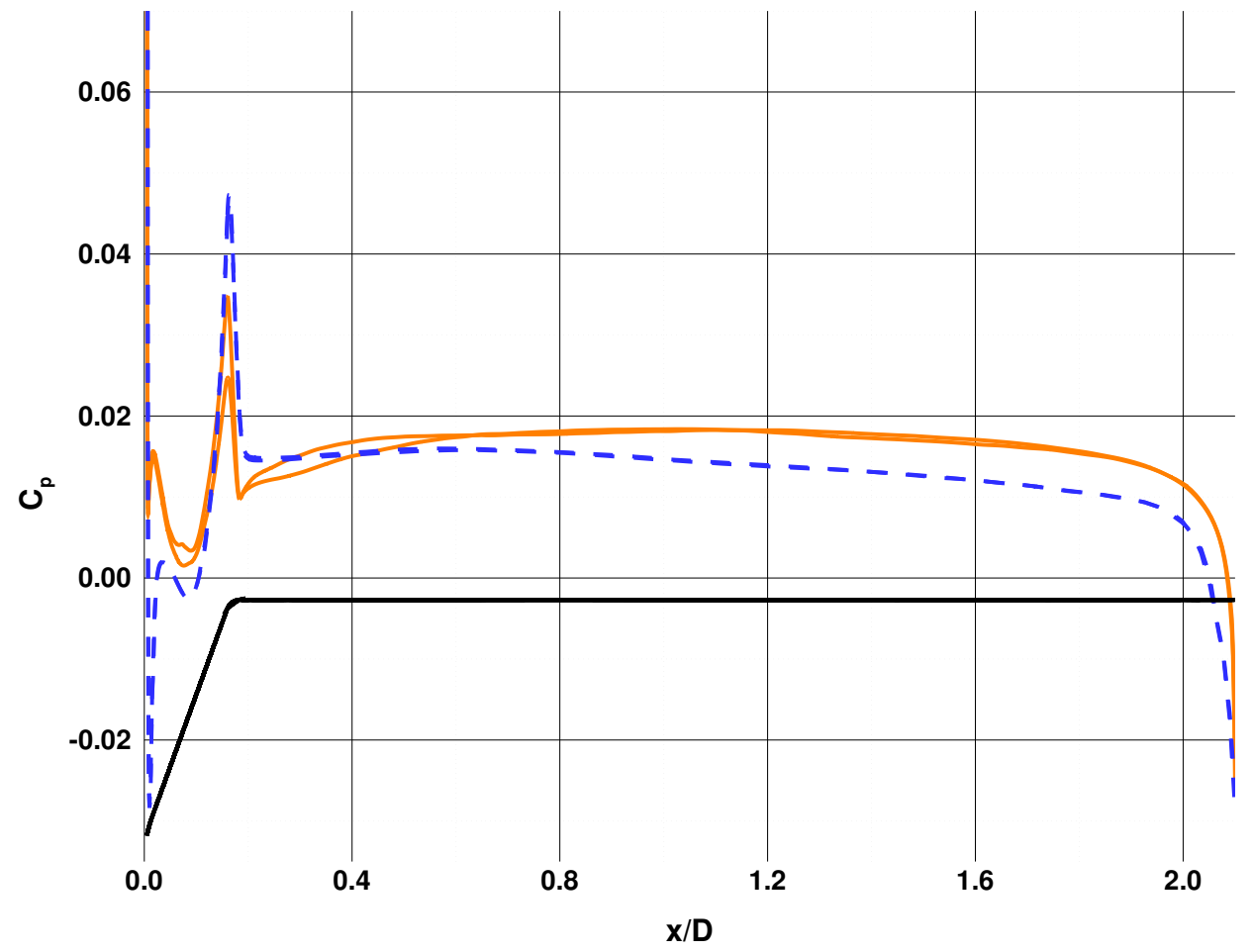

(b) Entire body.

Figure 21: Average surface pressure comparisons between Menter's SST turbulence model and SA-DES for Run 165, $\alpha=0^{\circ}$ (Fun3D). 


\section{Preliminary Comparisons}

Given that the uncertainty analysis for the validation experiment was not complete at the time of writing, this section will concentrate on code-to-code comparisons but will also include some preliminary experimental data without uncertainty estimates or corrections for wind tunnel flow angle, bias errors, or random errors. Three forms of comparisons will be made in this section: grid spacing distributions used by each code, prediction of the salient flowfield features and the experimentally observed frequency of oscillation, and finally a comparison of time-averaged surface pressures with raw experimental surface pressure data.

The first comparison is the grid spacing distributions used by each code. Figure 22 depicts a grid slice in the pitch plane focused on the jet interaction region. To be able to compare relative spacings between the grids even though Fun3D's tetrahedral grid is sliced into triangles and quadrilateral faces, the slice is colored by an isotropic spacing, $h$, which is the cube root of the cell volume. Each grid has clustering around the jet interaction area with DPLR having the finest with 0.005 diameter spacing, followed by OvERFLOw, Us3D, and Fun3D.

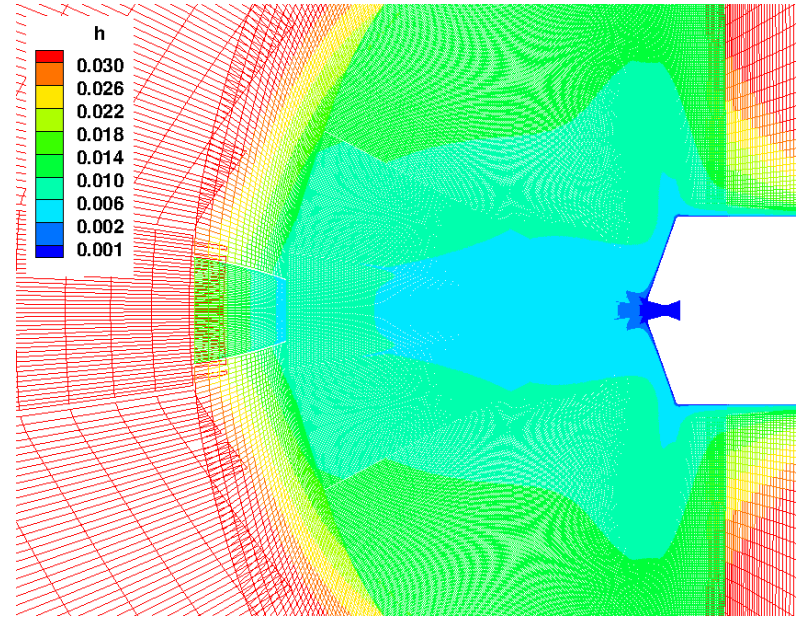

(a) DPLR.

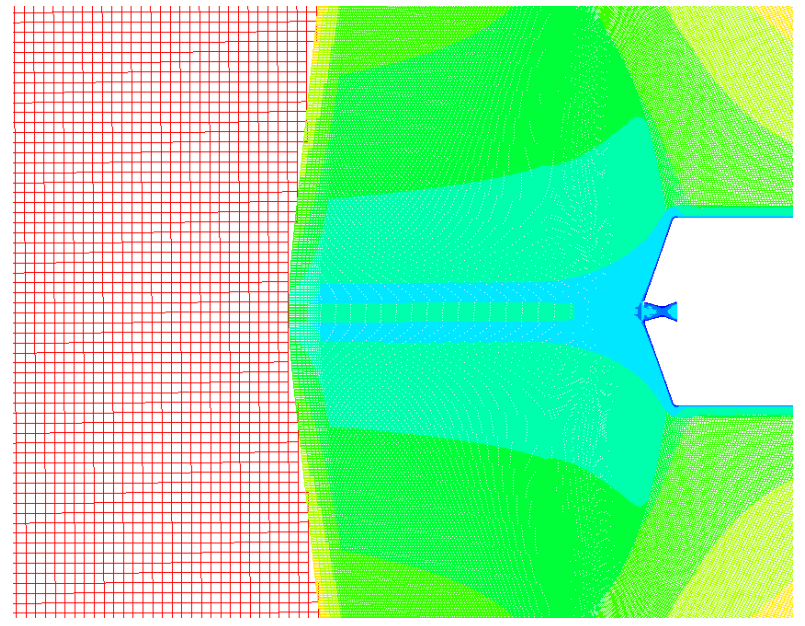

(c) Overflow.

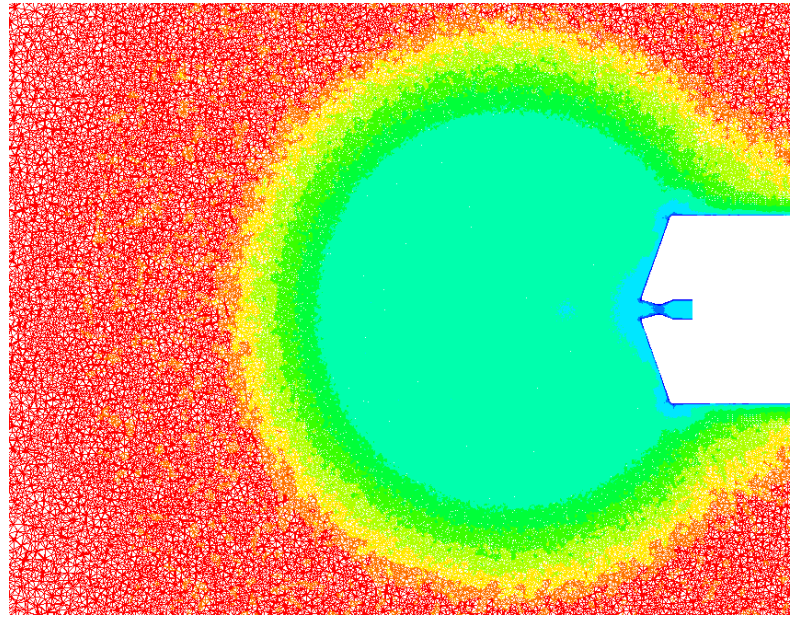

(b) Fun3D.

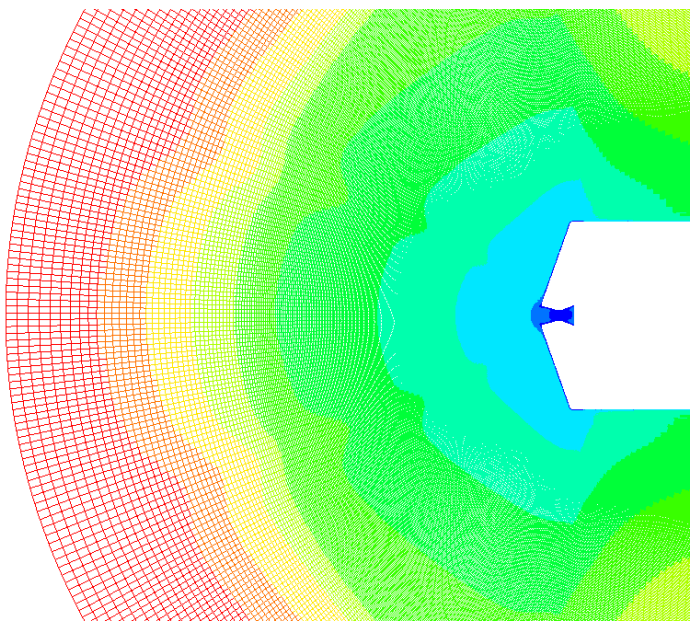

(d) Us3D.

Figure 22: Grid spacing distributions in the symmetry plane where $h$ is defined as (cell volume $)^{1 / 3}$. 
Figure 23 shows the forebody surface grid used by each code, where $h$ is now defined as the square root of the cell area. Here the distributions are more varied with the DPLR grid having more resolution around the jet exit than other grids but considerably less in the shoulder region. The Fun3D grid appears to have considerably more shoulder refinement than the others, but this an artifact of $h$ in that FUN3D's grid is isotropic and the rest have circumferentially-stretched anisotropic cells. On the forebody, OvERFLOw has the smallest spacing, followed by DPLR, Fun3D, and Us3D.

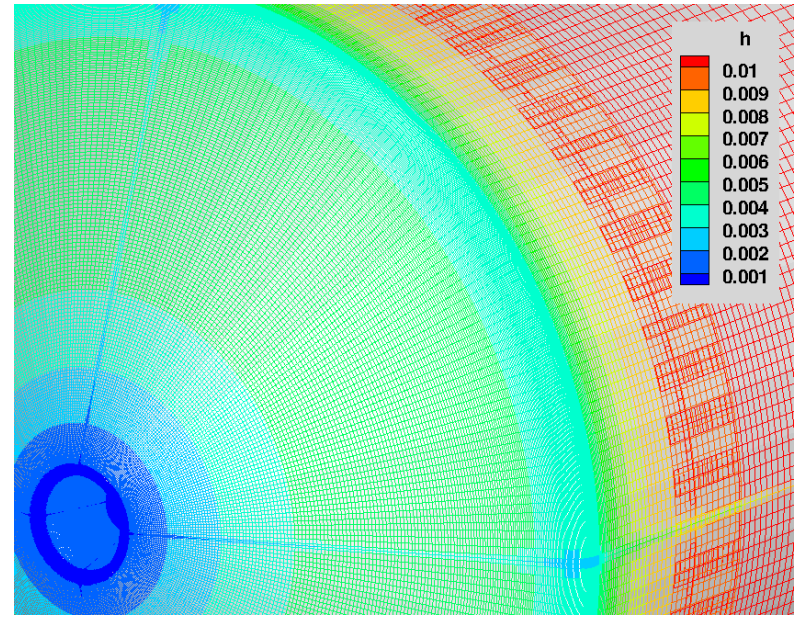

(a) DPLR.

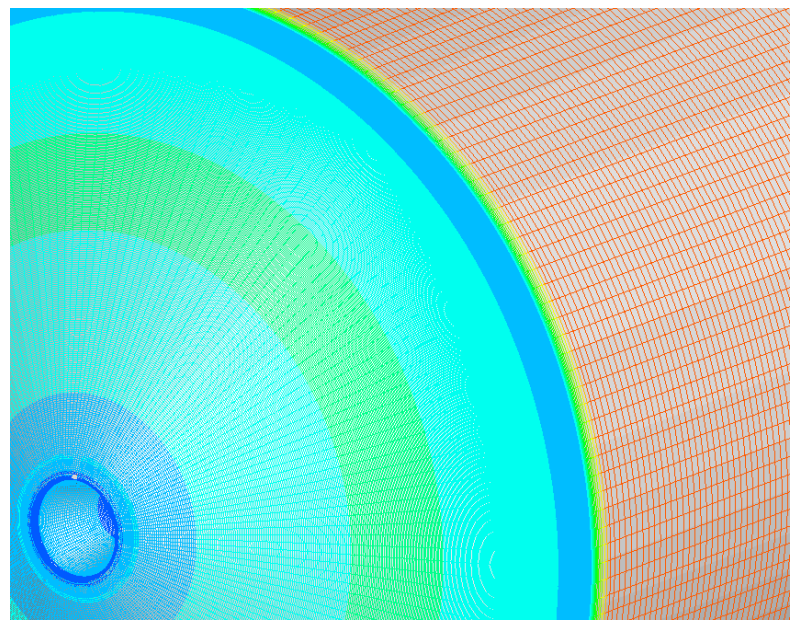

(c) Overflow.

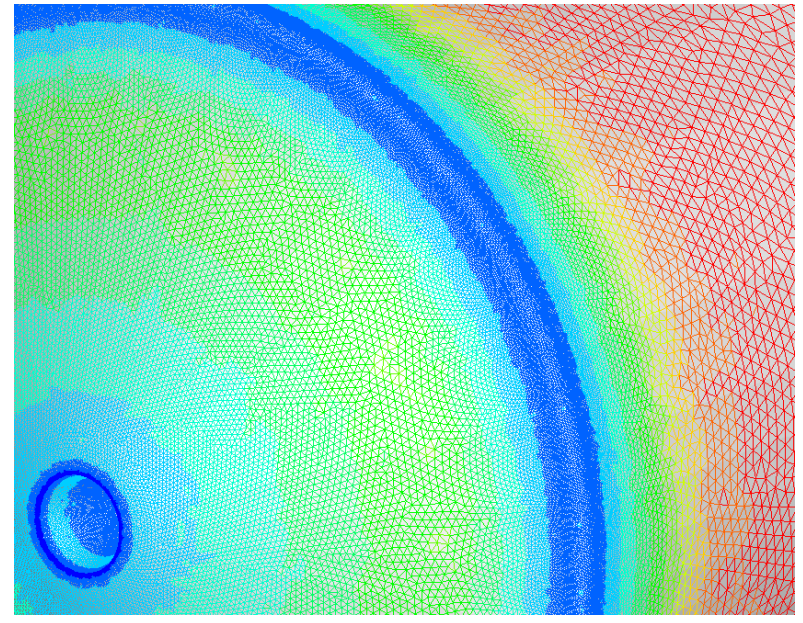

(b) Fun3D.

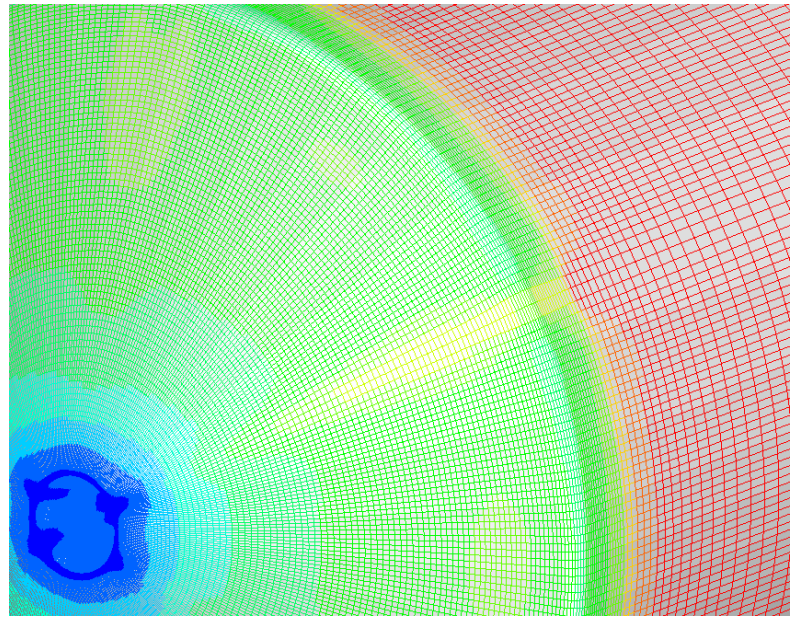

(d) Us3D.

Figure 23: Forebody surface grid for each code colored by $h$, which is (Cell Area $)^{1 / 2}$.

Figures 24 to 27 on pages 22-25 contain animations meant to simulate the experimental Schlieren movie shown in Figure 3a on page 5. DPLR and Us3D show Mach contours in the pitch plane, Fun3D has a simulated Schlieren via integrated density deflections through the computational volume similar to Ref. 37, and OvERFLOW presents a simulated focused shadowgraph via density gradients in the pitch plane. While all four codes capture the periodic vortex ring shedding from the tripoint region, the amplitude of the oscillation is considerably smaller for DPLR, which is the only code running the Menter's SST turbulence model-the others were run with DES, which does not inject turbulent eddy viscosity in this region. 


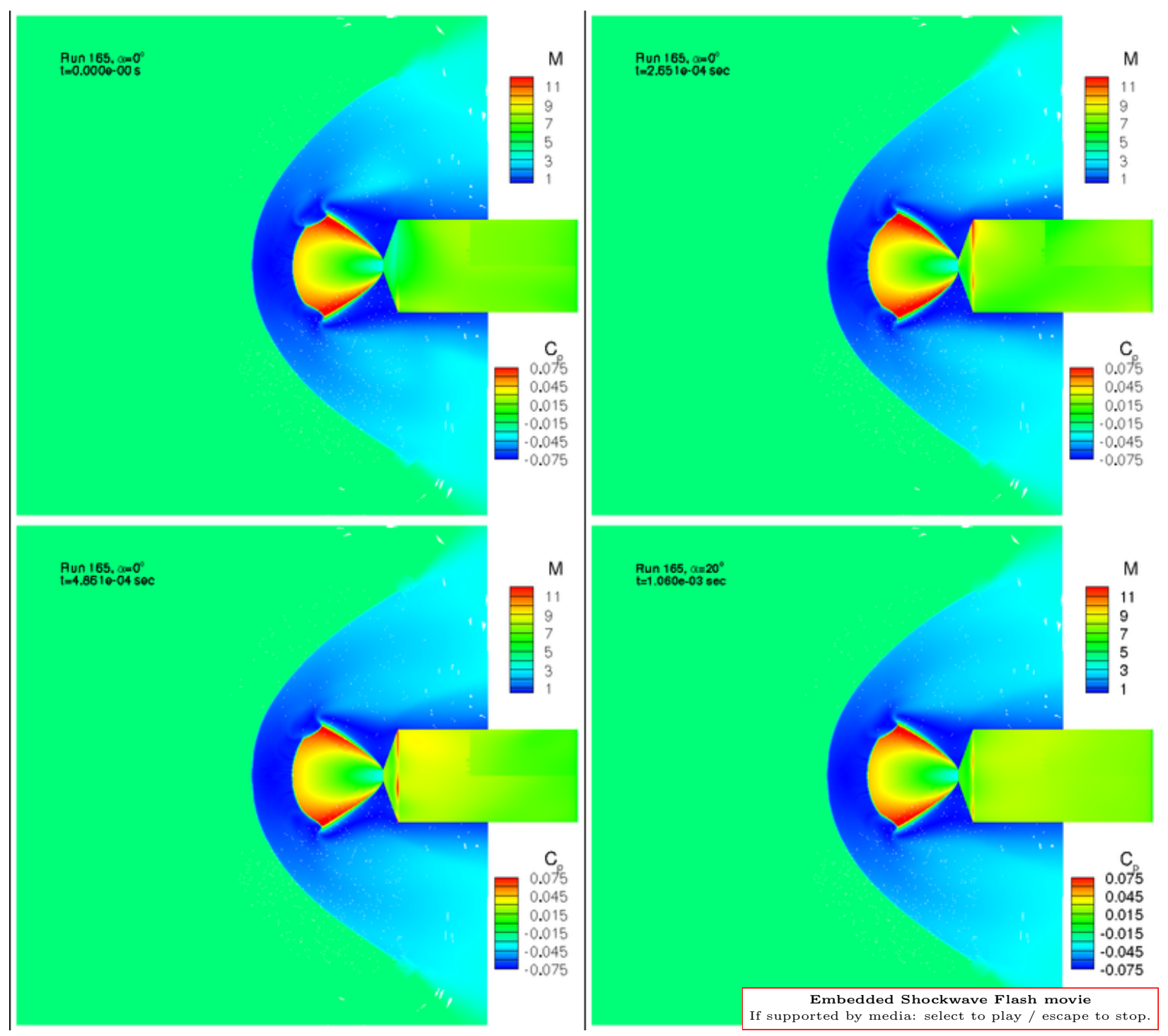

Figure 24: DPLR animation of symmetry plane Mach contours and surface $C_{p}$ for Run $165, \alpha=0^{\circ}$. The static image shows a tableau of four representative still frames. 


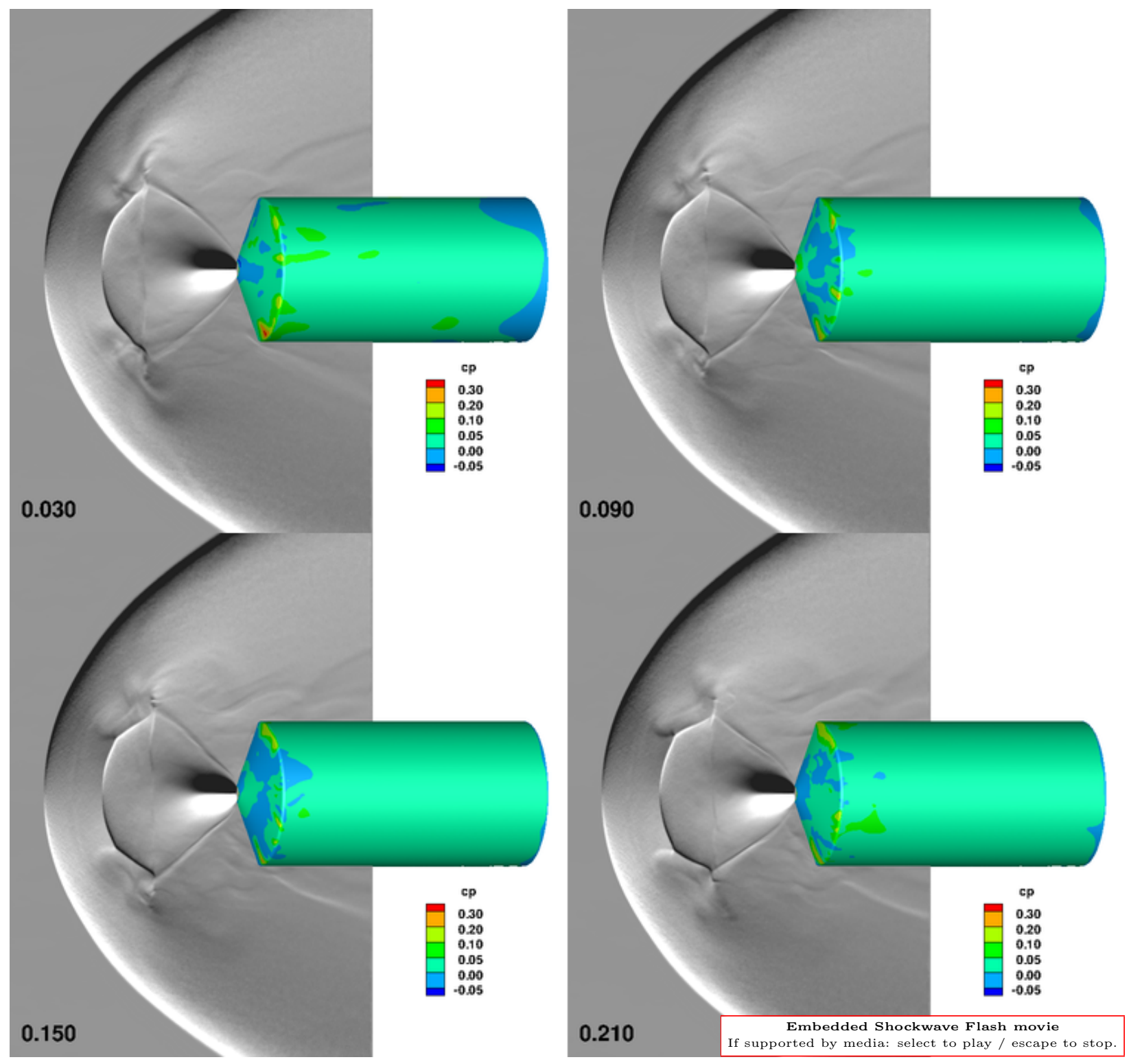

Figure 25: Fun3D-simulated Schlieren and surface $C_{p}$ animation for Run $165, \alpha=0^{\circ}$. The static image shows a tableau of four representative still frames. 


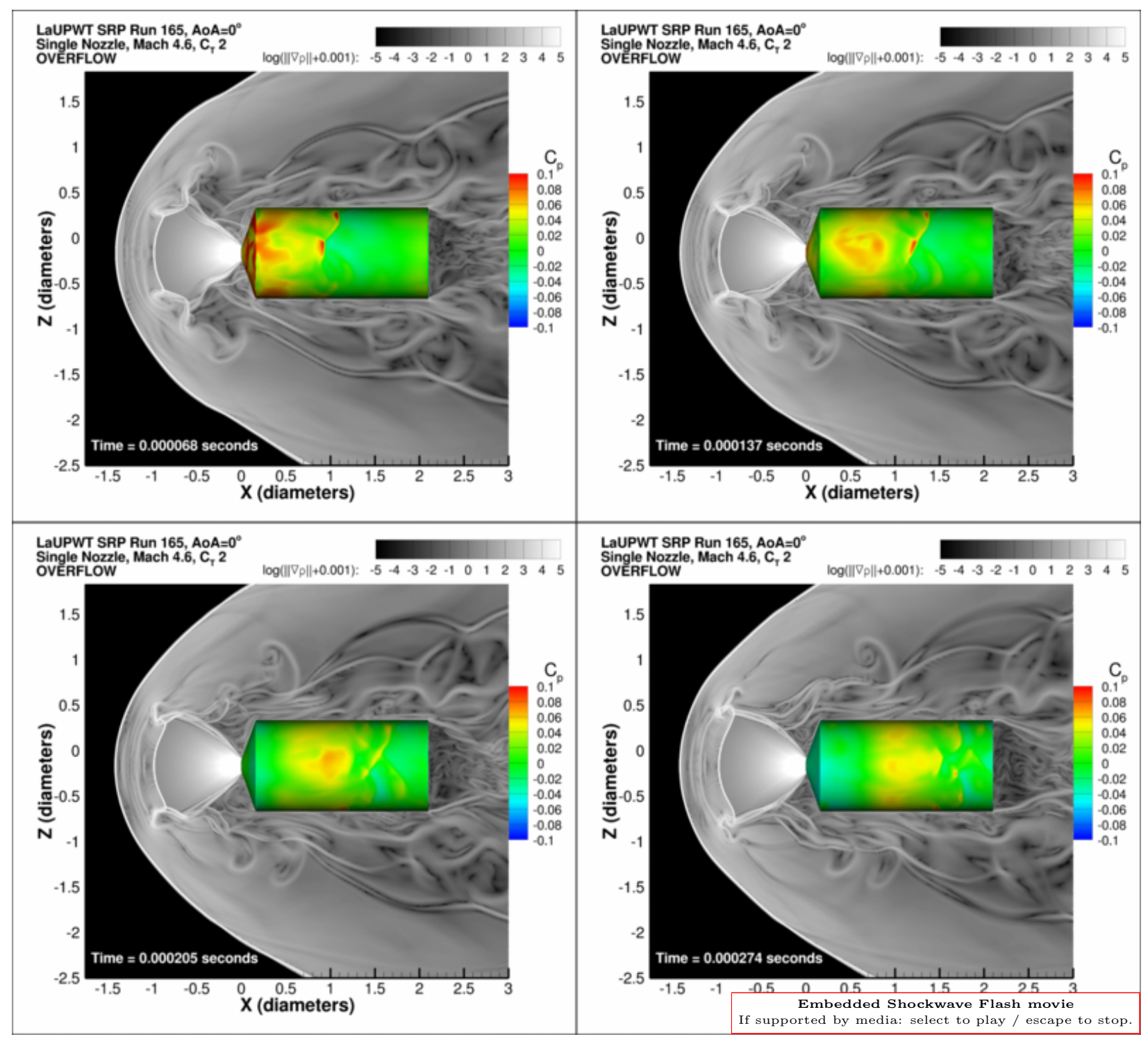

Figure 26: OvERfLOW animation of symmetry plane density gradients and surface $C_{p}$ for Run $165, \alpha=0^{\circ}$. The static image shows a tableau of four representative still frames. 


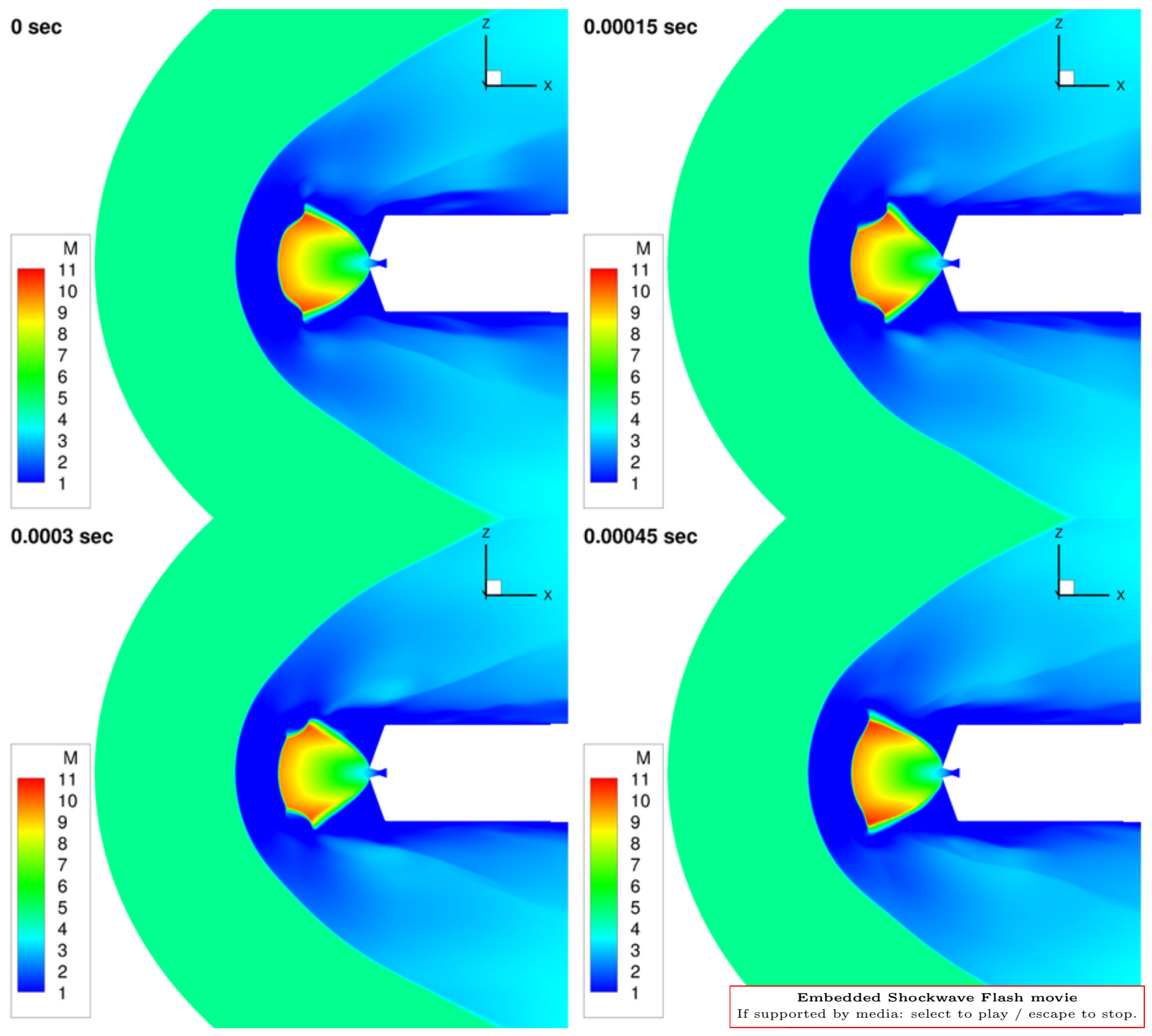

Figure 27: Us3D animation of symmetry plane Mach contours for Run 165, $\alpha=0^{\circ}$. The static image shows a tableau of four representative still frames. 
High-speed pressure transducers were post-processed to obtain root-mean-square (RMS)-averaged fluctuations and a power spectral density function for comparison with computations. ${ }^{17}$ For the zero angle of attack point of Run 165, the ring vortex shedding frequency, which is the dominant oscillation of the jet plume structure, is given in Table 4 along with all four codes plus an inviscid run by CART3D. ${ }^{48}$ As discussed in Ref. 17, depending on where the pressure transducer was located on the forebody, the predominant frequency ranged between $1.7 \mathrm{kHz}$ and $2.3 \mathrm{kHz}$. This range appears to correlate to a non-zero angle-of-attack due to tunnel flow angle. The frequency for DPLR and Us3D were obtained by performing a Fast Fourier Transform (FFT) of pressure at several forebody tap locations. CART3D computed the frequency via FFT of the axial force while OvERFLOW and FUN3D measured the steps per cycle of the axial force. All codes predict a frequency within the range of the experiment results; and as indicated by the inviscid CART3D result, the ring vortex shedding is captured inviscidly.

Figure 28 shows the comparison of time-averaged surface pressures between codes and the raw experimental data from Ref. 17 for Run 165, $\alpha=0^{\circ}$. Only the top and bottom centerline data are shown $\left(\theta=0^{\circ}, 180^{\circ}\right)$. Subfigure 28 a shows $C_{p}$ on the forebody as a function of radius from the jet exit at $r / R=0.1$ to the shoulder at $r / R=1$ while subfigure 28b spans the entire length of the model as a function of $x / D$. The raw experimental data shows some asymmetry on the forebody and significant asymmetry along the side body. As mentioned previously, this is thought to be due to an approximately one degree tunnel flow angle. Regardless, the $C_{p}$ levels for this zero angle-of-attack point are quite low as a result of the jet plume blocking the incoming flow and enveloping the entire model in subsonic recirculation regions; and so, some spread is anticipated on this expanded scale.

All codes predict similar trends for the forebody pressure distribution, however, the amount of pressure recovered on the forebody appears to correlate to grid resolution. As shown in Figure 22 on page 20 and Figure 23 on page 21, OvERFLow has the greatest concentration of points on and around the forebody and also predicts the highest pressure recovery on the forebody. Despite having relatively fine spacing in the forebody area compared to the grids used for FUN3D and Us3D, DPLR has a diminished peak near the jet exit. This is attributed to the SST turbulence model dissipating the periodically-shed ring vortex as shown in Figure 24 on page 22. Both Fun3D and Us3D ran SA-DES and agree well on the cone flank. Fun3D has a lower peak near the jet exit while Us3D has a lower peak at the shoulder, which correspond to where each has fewer grid points compared to the other.

The side-body pressure levels are similar for all codes run with DES while DPLR is slightly above the rest. Again, this may correspond to use of Menter's SST turbulence model. Note: The Us3D surface pressure does not drop near the base because the computational domain ends at the base plane.

Figure 29 on page 28 shows a similar comparison for the $12^{\circ}$ angle-of-attack point. Due to angle of attack, the pressures are markedly higher than those for the zero degree case and the deviations in the experimental data along a given upper or lower cut are not as pronounced. Similar to the zero degree angle-of-attack point and consistent with Figure 21 on page 19, the DES solutions (FUn3D, OvERFLOw, and Us3D) predict higher pressure recovery on the lower flank of the forebody than the pure RANS solution (DPLR). 


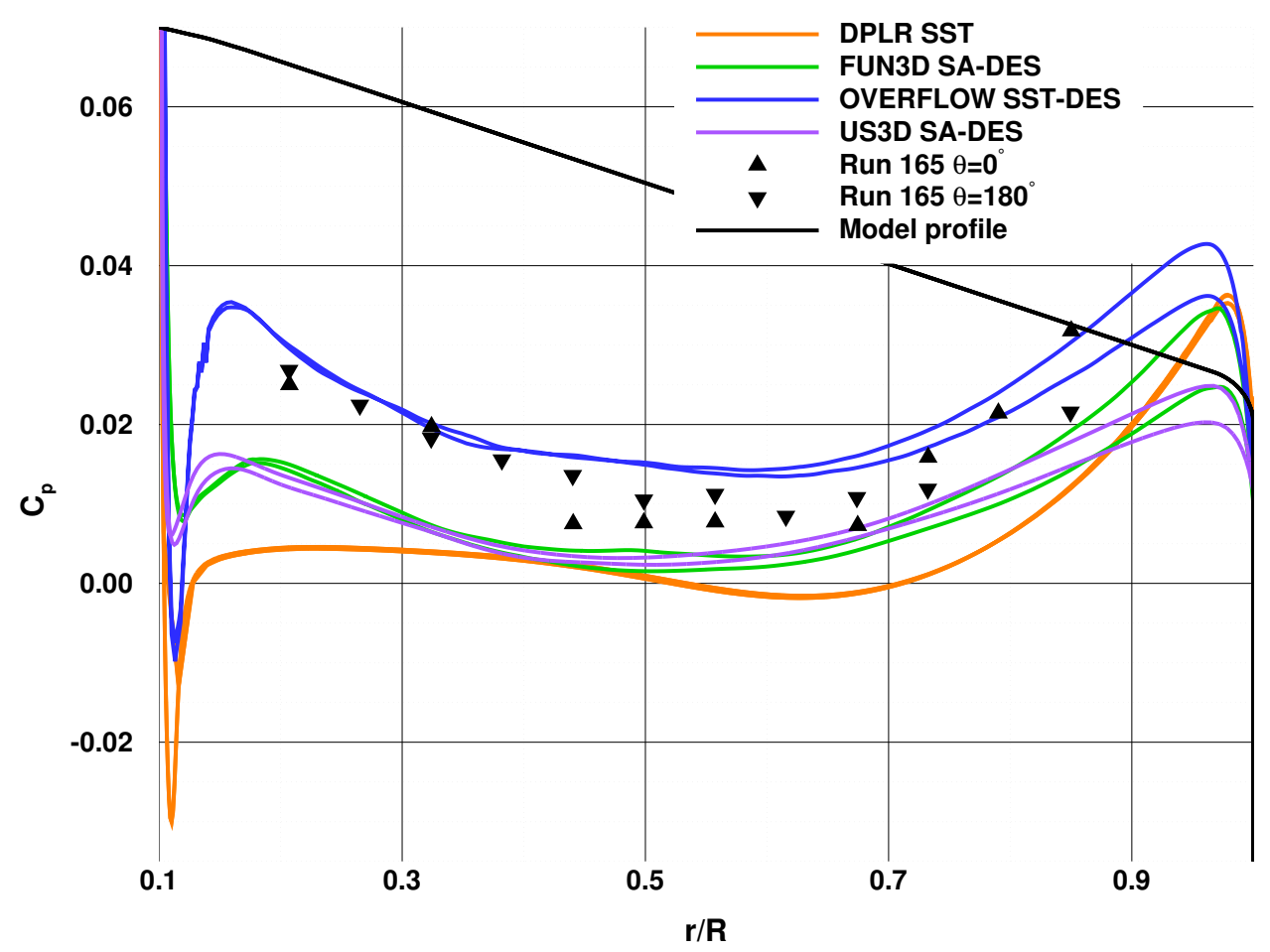

(a) Forebody only.

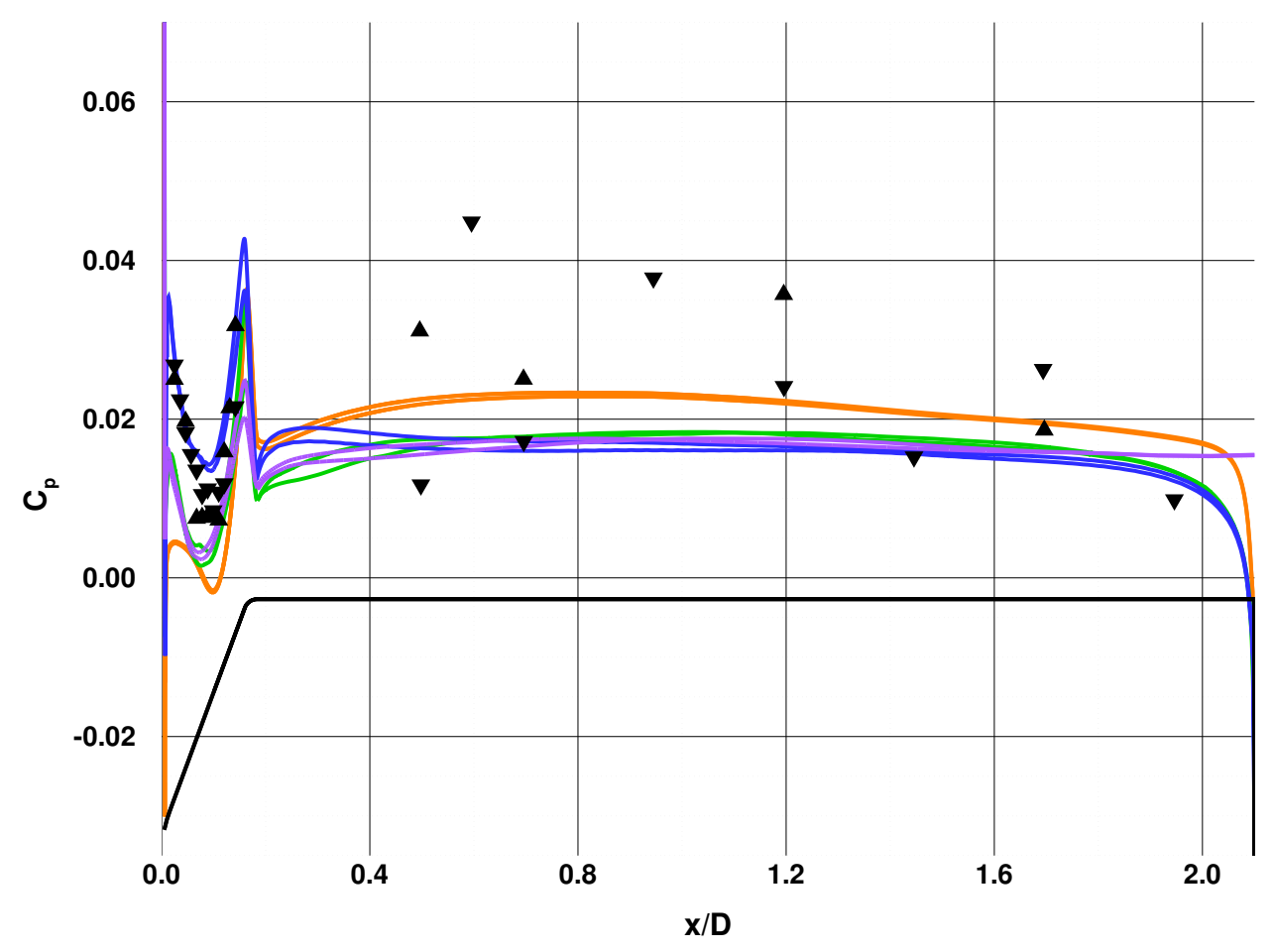

(b) Entire body.

Figure 28: Time-averaged surface pressure comparisons for Run 165, $\alpha=0^{\circ}$. 


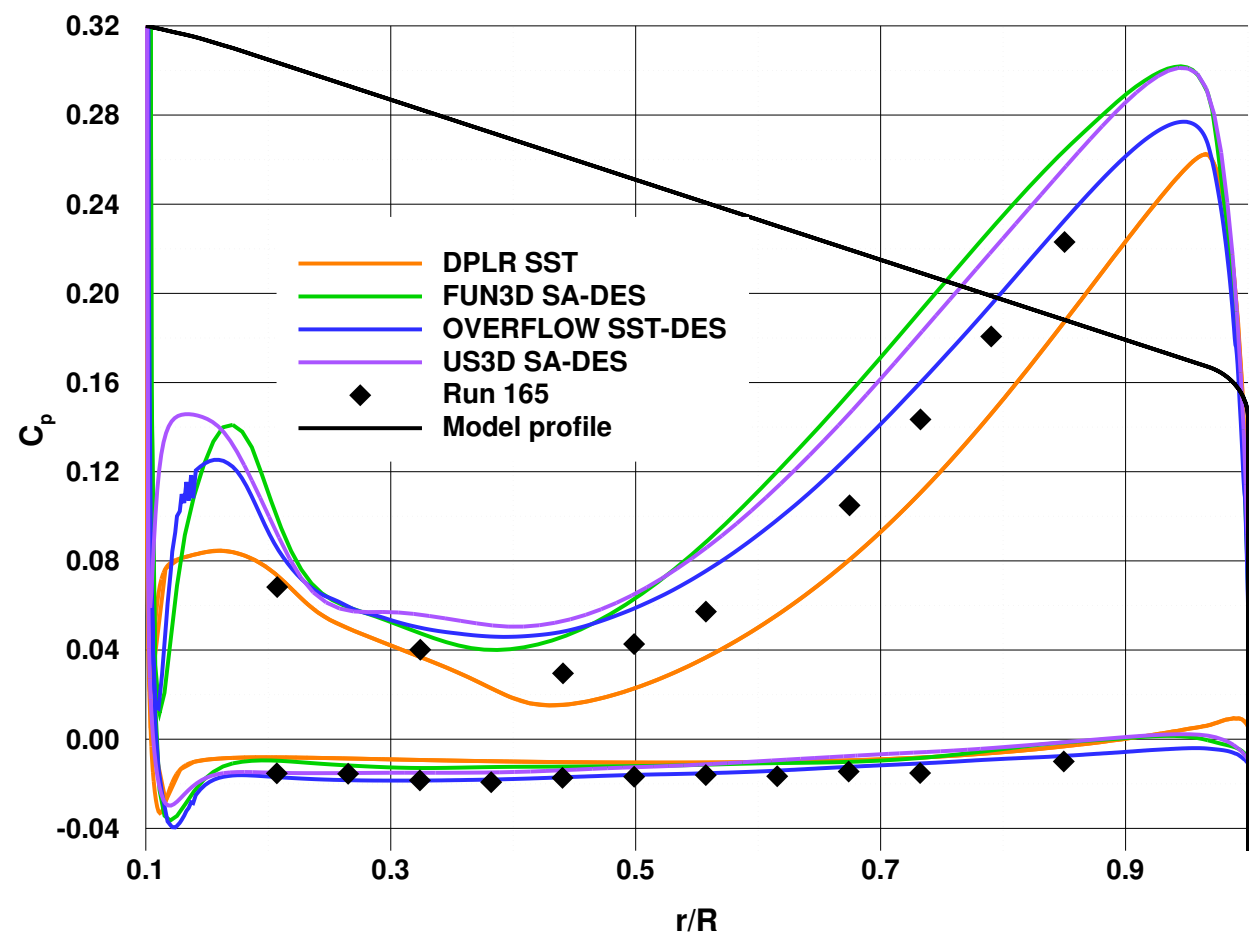

(a) Forebody only.

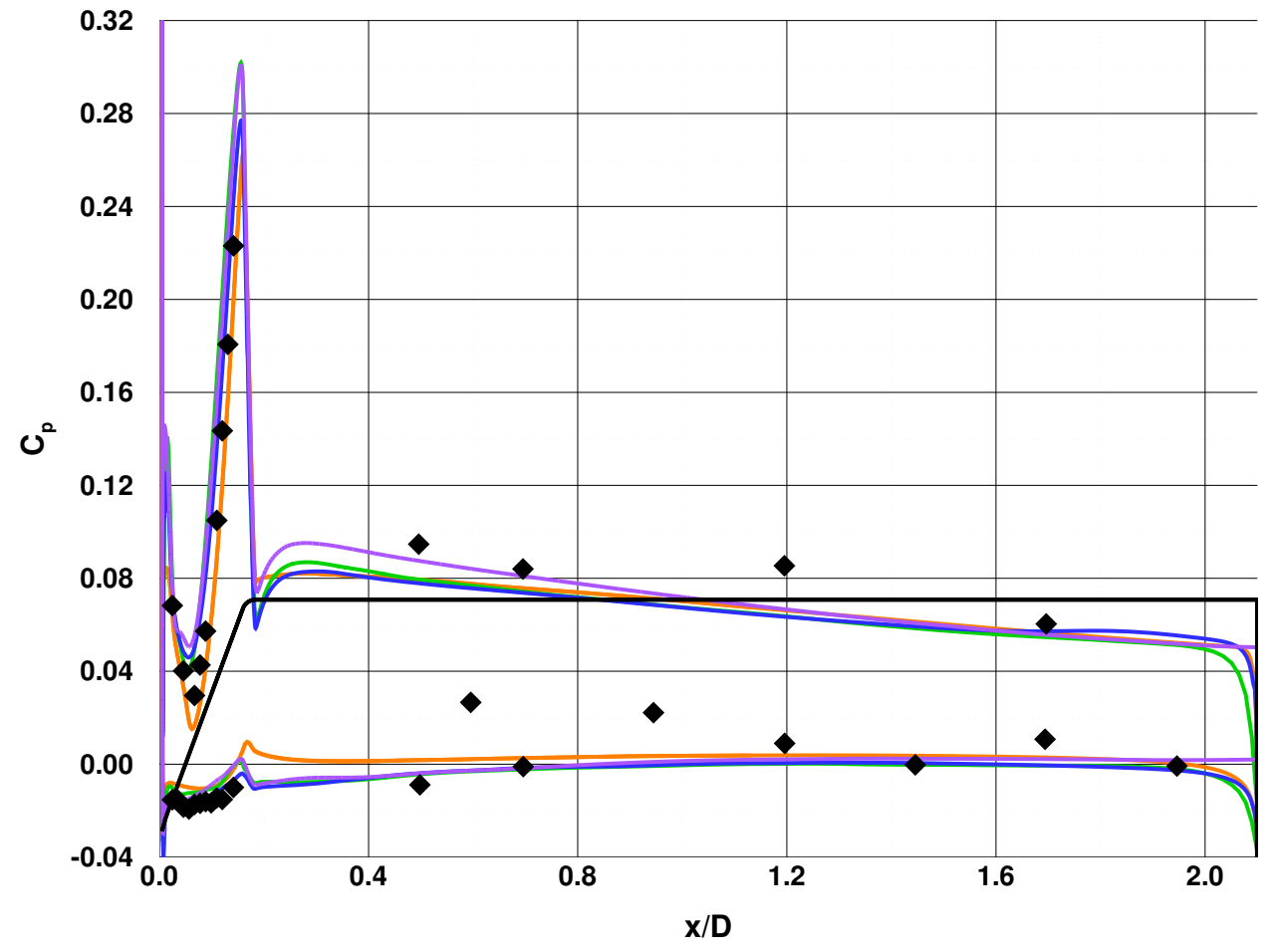

(b) Entire body.

Figure 29: Time-averaged surface pressure comparisons for Run $165, \alpha=12^{\circ}$. 


\section{Concluding Remarks}

In this paper we continued the process of verifying and validating computational fluid dynamics (CFD) codes for supersonic retropropulsive flows. In this installment, four CFD codes documented various numerical and physical modeling studies and made an initial comparisons with each other and with preliminary results from a wind tunnel experiment specifically designed to support CFD validation. Numerical studies varied in rigor from code-to-code comparisons to sensitivity studies to observed order-of-accuracy tests. Results indicate that for this complex, non-smooth flow, design order was not clearly observed. In addition, various physical modeling studies indicated that tunnel wall interference is minimal for the cases considered; the model mounting hardware effects are confined to the aft region of the model; and the RANS turbulence models damp or entirely dissipate the unsteadiness of this self-excited flow unless sufficient grid resolution and realizability constraints are used. To further resolve code-to-code differences, future work should consider running the same grid for multiple codes.

\section{Acknowledgments}

Phillip C. Stuart and Darby J. Vicker of NASA-Johnston Space Center, Houston, Texas; Thomas M. Booth Jacobs of Jacobs Technology, Houston, Texas; and Pieter Buning of NASA-Langley Research Center, Hampton, Virginia, provided guidance on gridding and solver best practices for Overflow. William T. Jones of NASA-Langley Research Center, Hampton, Virginia provided the GridEX/BatchEx unstructured grid generation framework and helped with its application to generate FUN3D grids. Todd R. White and Andrew J. Hyatt of ERC inc., Moffett Field, California helped DPLR develop best practices and provided overset gridding assistance for DPLR cases.

The authors would also like to acknowledge the support of the Exploration Technology Development and Demonstration (ETDD) Program, managed at NASA-Glenn Research Center. The work documented herein was performed as part of ETDD's Entry, Descent, and Landing (EDL) Technology Development Project, which is managed at NASA-Langley Research Center and supported by NASA-Ames Research Center, NASA-Johnson Space Center, and the Jet Propulsion Laboratory.

\section{References}

\footnotetext{
${ }^{1}$ Braun, R. D. and Manning, R. M., "Mars Exploration Entry, Descent, and Landing Challenges," Journal of Spacecraft and Rockets, Vol. 44, No. 2, Mar-Apr 2007.

${ }^{2}$ Korzun, A. M., Braun, R. D., and Cruz, J. R., "Survey of Supersonic Retropropulsion Technology for Mars Entry, Descent, and Landing," Journal of Spacecraft and Rockets, Vol. 46, No. 5, Sep-Oct 2009.

${ }^{3}$ Steinfeldt, B. A., Theisinger, J. E., Korzun, A. M., Clark, I. G., Grant, M. J., and Braun, R. T., "High Mass Mars Entry, Descent, and Landing Architecture Assessment," AIAA Paper 2009-6684, Sept. 2009.

${ }^{4}$ Zang, T. A., Dwyer-Dianciolo, A. M., Kinney, D. J., Howard, A. R., Chen, G. T., Ivanov, M. C., Sostaric, R. R., and Westhelle, C. H., "Overview of the NASA Entry, Descent and Landing Systems Analysis Study," AIAA Paper 2010-8649, Aug. 2010.

${ }^{5}$ Edquist, K. T., Dyakonov, A. A., Korzun, A. M., Shidner, J. D., Studak, J. W., Tigges, M. A., Kipp, D. M., Prakash, R., Trumble, K. A., and Dupzyk, I. C., "Development of Supersonic Retro-Propulsion for Future Mars Entry, Descent, and Landing Systems," AIAA Paper 2010-5046, Jun 2010.

${ }^{6}$ Oberkampf, W. L. and Roy, C., Verification and Validation in Scientific Computing, Cambridge University Press, Cambridge, MA, 2010.

${ }^{7}$ Aeschliman, D. P. and Oberkampf, W. L., "Experimental Methodology for Computational Fluid Dynamics Code Validation," AIAA Journal, Vol. 36, No. 5, May 1998, pp. 733-741.

${ }^{8}$ Jarvinen, P. O. and Adams, R. H., "The Aerodynamic Characteristics of Large Angled Cones with Retrorockets," NASA Contractor Report NAS7-576, Feb. 1970.

${ }^{9}$ McGhee, R., "Effects of a Retronozzle Located at the Apex of a $140^{\circ}$ Blunt Cone at Mach Numbers of 3, 4.5, and 6," NASA TN D-6002, Jan. 1971.

${ }^{10}$ Daso, E. O., Pritchett, V. E., Wang, T. S., Ota, D. K., Blankson, I. M., and Auslender, A. H., "Dynamics of Shock Dispersion and Interactions in Supersonic Freestream with Counterflowing Jets," AIAA Journal, Vol. 47, No. 6, June 2009, pp. 1313-1326.

${ }^{11}$ Trumble, K. A., Schauerhamer, D. G., Kleb, W. L., Carlson, J.-R., Buning, P. G., Edquist, K. T., and Barnhardt, M. D., "An Initial Assessment of Navier-Stokes Codes Applied to Supersonic Retro-Propulsion," AIAA Paper 2010-5047, June 2010.

${ }^{12}$ Korzun, A. M., Cordell, Jr., C. E., and Braun, R. D., "Comparison of Inviscid and Viscous Aerodynamic Predictions of Supersonic Retropropulsion Flowfields," AIAA Paper 2010-5048, June 2010.

${ }^{13}$ Cheng, G. C., Neroorkar, K. D., Chen, Y. S., Wang, T. S., and Daso, E. O., "Numerical Study of Flow Augmented Thermal Management for Entry and Re-Entry Environments," AIAA Paper 2007-4560, June 2007.
} 
${ }^{14}$ Cordell, Jr., C. E., Clark, I. G., and Braun, R. D., "CFD Verification of Supersonic Retropropulsion for a Central and Peripheral Configuration," IEEE Aerospace Conference Paper 1190, March 2011.

${ }^{15}$ Bakhtian, N. M. and Aftosmis, M. J., "Parametric Study of Peripheral Nozzle Configurations for Supersonic Retropropulsion," Journal of Spacecraft and Rockets, Vol. 47, No. 6, November-December 2010, pp. 935-950.

${ }^{16}$ Berry, S. A., Laws, C. T., Kleb, W. L., Rhode, M. N., Spells, C., McCrea, A. C., Trumble, K. A., Schauerhamer, D. G., and Oberkampf, W. L., "Supersonic Retro-Propulsion Experimental Design for Computational Fluid Dynamics Model Validation," IEEE Aerospace Conference Paper 1499, March 2011.

${ }^{17}$ Berry, S. A., Rhode, M. N., Edquist, K. T., and Player, C. J., "Supersonic Retropropulsion Experimental Results from the NASA Langley Unitary Plan Wind Tunnel," AIAA Paper 2011-TBD, June 2011.

${ }^{18}$ Trumble, K. A., Schauerhamer, D. G., Kleb, W. L., Carlson, J.-R., and Edquist, K. T., "Analysis of Navier-Stokes Codes Applied to Supersonic Retro-Propulsion Wind Tunnel Test," IEEE Aerospace Conference Paper 1471, March 2011.

${ }^{19}$ Wright, M. W., White, T., and Mangini, N., "Data Parallel Line Relaxation (DPLR) Code User Manual Acadia Version 4.01.1," NASA/TM 2009-215388, Oct. 2009.

${ }^{20}$ Anderson, W. K. and Bonhaus, D. L., "An Implicit Upwind Algorithm for Computing Turbulent Flows on Unstructured Grids," Computers and Fluids, Vol. 23, No. 1, 1994, pp. 1-21.

${ }^{21}$ Anderson, W. K., Rausch, R. D., and Bonhaus, D. L., "Implicit/Multigrid Algorithms for Incompressible Turbulent Flows on Unstructured Grids," Journal of Computational Physics, Vol. 128, 1996, pp. 391-408.

${ }^{22}$ Buning, P. G., Jespersen, D. C., Pulliam, T. H., Klopfer, G. H., Chan, W. M., Slotnick, J. P., Krist, S. E., and Renze, K. J., "Overflow Users Manual," Tech. rep., NASA Langley Research Center, 2002.

${ }^{23}$ Nompelis, I. N., Drayna, T., and Candler, G. V., "A Parallel Unstructured Implicit Solver for Hypersonic Reacting Flow Simulations," AIAA Paper 2005-4867, June 2005.

${ }^{24}$ Jr., C. M. J., Corlett, W. A., and Monta, W. J., "Description and Calibration of the Langley Unitary Plan Wind Tunnel," NASA TP 1905, Nov. 1981.

${ }^{25}$ Roy, C. J., "Review of Code and Solution Verification Procedures for Computational Simulation," Journal of Computational Physics, Vol. 205, 2005, pp. 131-156.

${ }^{26}$ MacCormack, R. W. and Candler, G. V., "The Solution of the Navier-Stokes Equations Using Gauss-Seidel Line Relaxation," Computers and Fluids, Vol. 17, No. 1, 1989, pp. 135-150.

${ }^{27}$ van Leer, B., "Towards the Ultimate Conservative Scheme. V. A Second-Order Sequel to Godunov's Method," Journal of Computational Physics, Vol. 32, 1979, pp. 101-136.

${ }^{28}$ Yee, H. C., "A Class of High-Resolution Explicit and Implicit Shock Capturing Methods," NASA TM 101088, Feb. 1989.

${ }^{29}$ Menter, F. R., "Improved Two-Equation $k-\omega$ Turbulence Models for Aerodynamic Flows," NASA TM 103975, Oct. 1992.

${ }^{30}$ Roy, C. J., "Grid Convergence Error Analysis for Mixed-Order Numerical Schemes," AIAA Journal, Vol. 41, No. 4, April 2003, pp. 595-604.

${ }^{31}$ Edwards, J. R., "A Low-Diffusion Flux-Splitting Scheme for Navier-Stokes Calculation," Computers Es Fluids, Vol. 26, 1997, pp. 653-659.

${ }^{32}$ van Albada, G. D., van Leer, B., and Roberts, W. W., "A Comparative Study of Computational Methods in Cosmic Gas Dynamics," Astronomy \& Astrophysics, Vol. 108, 1982, pp. 76.

${ }^{33}$ Vatsa, V. N. and White, J. A., "Calibration of a Unified Flux Limiter for Ares-Class Launch Vehicles from Subsonic to Supersonic Speeds," JANNAF paper, April 2009.

${ }^{34}$ Vatsa, V. N. and Lockard, D. P., "Assessment of Hybrid RANS/LES Turbulence Models for Aeroacoustics Applications," AIAA Paper 2010-4001, June 2010.

${ }^{35}$ Vatsa, V. N., Carpenter, M. H., and Lockard, D. P., "Re-evaluation of an Optimized Second Order Backward Difference (BDF2OPT) Scheme for Unsteady Flow Applications," AIAA Paper 2010-0122, Jan. 2010.

${ }^{36}$ Thomas, J. L., Diskin, B., and Rumsey, C. L., "Towards Verification of Unstructured-Grid Solvers," AIAA Journal, Vol. 46, No. 2, jan 2008, pp. 3070-3079.

${ }^{37}$ Yates, L. A., "Interferograms, Schlieren, and Shadowgraphs Constructed from Real- and Ideal-Gas, Two- and ThreeDimensional Computed Flowfields," NASA CR 190054, Jan. 1992.

${ }^{38}$ Tramel, R., Nichols, R., and Buning, P. G., "Addition of Improved Shock-Capturing Schemes to OVERFLOW 2.1," AIAA Paper 2009-3988, June 2009.

${ }^{39}$ Menter, F. R., "Two-Equation Eddy-Viscosity Turbulence Models for Engineering Applications," AIAA Journal, Vol. 32, No. 8, 1994, pp. 1598-1605.

${ }^{40}$ Wilcox, D. C., "Formulation of the $k-\omega$ Turbulence Model Revisited," AIAA Journal, Vol. 46, No. 11, Nov. 2008, pp. $2823-2838$.

${ }^{41}$ Nichols, R. H., Tramel, R. W., and Buning, P. G., "Solver and Turbulence Model Upgrades to OVERFLOW 2 for Unsteady and High-Speed Applications," AIAA Paper 2006-2824, June 2006.

${ }^{42}$ Nompelis, I. N., Drayna, T., and Candler, G. V., "Development of a Hybrid Unstructured Implicit Solver for the Simulation of Reacting Flows Over Complex Geometries," AIAA Paper 2004-2227, Jan. 2004.

${ }^{43}$ Spalart, P. R. and Allmaras, S. R., "A One-Equation Turbulence Model for Aerodynamic Flows," AIAA Paper 92-0439, Jan. 1992.

${ }^{44}$ Spalart, P. R., Jou, W.-H., Strelets, M., and Allmaras, S. R., "Comments on the Feasibility of LES for Wings and on a Hybrid RANS/LES Approach," Afosr international conference on dns/les, 1997.

${ }^{45}$ Spalart, P. R., Deck, S., Shur, M. L., Squires, K. D., Strelets, M. K., and Travin, A., "A new version of detached-eddy simulation, resistant to ambiguous grid densities," Theor. Comput. Fluid Dyn., Vol. 20, May 2006, pp. 181-195.

${ }^{46}$ Sánchez-Rocha, M. and Menon, S., "The compressible hybrid RANS/LES formulation using an additive operator," Journal of Computational Physics, Vol. 228, Dec. 2008, pp. 2037-2062. 
${ }^{47}$ Suzen, Y. B. and Hoffmann, K. A., "Investigation of Supersonic Jet Exhaust Flow by One- and Two-Equation Turbulence Models," AIAA Paper 98-0322, Jan. 1998.

${ }^{48}$ Bakhtian, N. M. and Aftosmis, M. J., "Analysis of Inviscid Simulations for the Study of Supersonic Retropropulsion," AIAA Paper 2011-TBD, June 2011. 